\title{
THE FAUNA OF CAVES AND OTHER SUBTERRANEAN HABITATS OF NORTH CAROLINA, USA
}

\author{
Cato Holler Jr. ${ }^{1}$, Jonathan D. Mays², and Matthew L. Niemiller ${ }^{3, C}$
}

\begin{abstract}
Over 1,500 caves have been documented in North Carolina, however, cave fauna in the Blue Ridge Mountains and Piedmont regions of North Carolina have been overlooked historically compared to the cave-rich karst terrains in the Appalachian Valley and Ridge and Interior Low Plateau to the west. Here, we provide the first comprehensive faunal list of caves and other subterranean habitats in the state based on over 40 years of periodic surveys and compilation of literature, biodiversity databases, and museum records. We report 475 occurrences from 127 caves, springs, and wells in 29 counties, representing 5 phyla, 17 classes, 43 orders, 90 families, 124 genera, and at least 164 species. Vertebrate fauna comprised 32 species, including 4 fishes, 9 salamanders, 1 lizard, 4 snakes, 2 birds, and 12 mammals ( 8 bats). Diverse invertebrate groups included spiders ( 11 families and 18 genera), springtails ( 7 families and 9 genera), segmented worms ( 3 families and 8 genera), and snails (6 families and 9 genera). At least 25 taxa are troglobites/ stygobites (cave obligates), including 5 species of cave flatworms, 5 cave springtails, and 5 cave amphipods. Most troglobitic/stygobitic fauna documented in this study are endemic to North Carolina. Counties with the greatest cave biodiversity include Rutherford, McDowell, Swain, Henderson, Polk, and Avery counties. Over 20 species documented are of conservation concern, including 14 troglobites and 3 federally-listed bats. Although not as diverse as adjacent states, caves and other subterranean habitats in North Carolina support a diverse community of invertebrates and vertebrates. Our review serves as a base line for future cave biological surveys in the state and highlights the importance of subterranean habitats for North Carolina biodiversity.
\end{abstract}

\section{Introduction}

Caves and associated subterranean habitats are home to a unique and taxonomically diverse assemblage of invertebrate and vertebrate organisms in North America. In addition to the more than 1,350 species that are obligate inhabitants of terrestrial and aquatic subterranean habitats in the United States (i.e., troglobites and stygobites) (Niemiller et al., 2019), hundreds of other species use caves on an occasional to semi-permanent basis. Most of this subterranean diversity is known from the ten major karst biogeographic regions defined in the United States that are associated with carbonate exposures (Culver et al., 2003; Hobbs, 2012). Several additional smaller karst regions exist, but these regions have received comparatively little attention from biospeleologists.

The fauna of caves and associated subterranean habitats in the Blue Ridge Mountains and Piedmont physiographic provinces of North Carolina has been grossly understudied compared to subterranean fauna of the cave-rich Appalachian and Interior Low Plateau karst to the west of the Appalachian Mountains in Tennessee, Alabama, Georgia, and Virginia. In contrast to most cave systems in the Appalachians and Interior Low Plateau karst regions, cave systems in North Carolina are predominately non-solutional (i.e., pseudo-karst) and granite-gneiss talus and fissure caves, in particular. A few troglobites and stygobites are known from North Carolina, including some that are endemic to the state, such as the Carolina Groundwater Amphipod (Stygobromus carolinensis) and Blowing Springs Cave Springtail (Pseudosinella flatua). The bat fauna from several caves and mines has been characterized (Boynton et al., 1992), particularly in association to winter hibernation surveys in recent years (e.g., North Carolina Wildlife Resources Commission, 2017b). However, a comprehensive, annotated list of the subterranean fauna of North Carolina has never been published.

In early 1972, after the Flittermouse Grotto of the National Speleological Society was chartered, its members initiated the North Carolina Cave Survey (NCCS) to track and catalog caves in the state. In conjunction with this statewide cave inventory, and with the suggestion and encouragement of the late Dr. John Cooper, former Director of Research and Collections at the North Carolina State Museum of Natural Sciences, a survey of North Carolina's little-known cave fauna also began. Here we report new distributional records of subterranean fauna documented during periodic surveys of caves between 1970 and 2018 conducted by the lead author and maintained by the NCCS. We also compiled occurrence records from caves and other subterranean habitats (e.g., wells, springs, and seeps) for invertebrate and vertebrate fauna of North Carolina from several sources, including published and unpublished literature, databases, and museum collections. We include these data to generate the first comprehensive faunal list from caves in the state.

\footnotetext{
${ }_{1}^{1}$ Director, North Carolina Cave Survey, PO Box 100, Old Fort, North Carolina 28762; hollers2@frontier.com

${ }^{2}$ Fish and Wildlife Research Institute, Florida Fish and Wildlife Conservation Commission, 1105 SW Williston Rd., Gainesville, Florida, 32601; jonathan.mays@myfwc.com

${ }^{3}$ Department of Biological Sciences, The University of Alabama in Huntsville, Huntsville, Alabama 35899; cavemander17@gmail.com, matthew. niemiller@uah.edu

${ }^{\mathrm{C} C o r r e s p o n d i n g ~ a u t h o r ~}$
} 


\section{Materials and Methods}

\section{STUDY REGION AND GEOLOGICAL SETTING}

North Carolina lies in the southeastern United States bordered on the east by the Atlantic Ocean, on the south by South Carolina and Georgia, on the west by Tennessee, and on the north by Virginia. The state is divided into three physiographic regions: i) the Blue Ridge Mountains, composed of assorted igneous, metamorphic, volcanic, and sedimentary rocks of widely ranging ages; ii) the Piedmont Plateau consisting primarily of metamorphic and igneous rocks, and iii) the extensive Coastal Plain with its sands, clays, phosphates, and limestone, making up 45\% of the state.

As of October 2018, the North Carolina Cave Survey (NCCS) has 1,582 caves on record (Figure 1). Some states limit the term "cave" to a specific length measure; for example, several state cave surveys require that a passage be at least 50 feet $(15.2 \mathrm{~m})$ in length to be considered a cave. Karst regions and solution caves are not common in North Carolina, thus the NCCS recognizes the term as any naturally occurring opening within the earth capable of being entered by a human. True karst (solution) caves are limited to a few areas of the state. Madison County has three cave-bearing geological formations: Shady Dolomite, the Honaker Limestone, and a dolomite member of the Rome formation. Several significant caves have been dissolved out of the Murphy Marble in Swain County. The Cambrian age Shady Dolomite also occurs in McDowell County where numerous caves have resulted, including Linville Caverns, a well-known commercial cave. Finally, on the Coastal Plain, Eocene age limestones harbor several small solutional caves.

What the state is lacking in karst is more than made up for in its pseudokarst. Most caves in North Carolina are, in fact, pseudokarstic in origin, created not by solution but by physical processes, such as gravity-sliding producing talus and fissure caves, often in igneous and metamorphic rocks (Holler, 2019). Several of these are surprisingly extensive. Bat Cave, for example, has over $2 \mathrm{~km}$ of surveyed passages and represents the longest known pseudokarst cave of its type (augen-gneiss) in the world (Holler, 1981; Holler and Holler, 2009).

\section{BIOLOGICAL INVENTORIES}

The lead author $(\mathrm{CH})$ conducted periodic biological inventories of caves and fissures in North Carolina from 1970 to 2018. Most of the cave surveys were in the western North Carolina counties of Avery, Burke, McDowell, Rutherford, and Swain. Bioinventories involved visual-encounter surveys for cave life in terrestrial, riparian, and aquatic habitats, such

\section{NORTH CAROLINA}

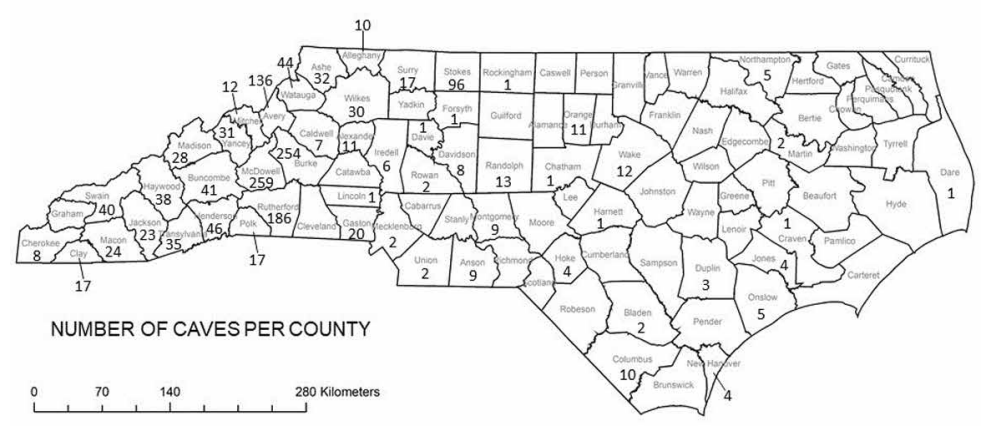

Figure 1. Distribution of the 1,582 caves by county in North Carolina. Data from the North Carolina Cave Survey (NCCS) as of October 2018. as entrance areas, cave walls and ceilings, mud banks, rimstone pools, and cave streams. These surveys systematically traversed the cave from the entrance to the farthest extent of the cave explorable by the research team. Search effort included lifting rocks and other cover, as well as searching through cobbles, detritus, and organic matter. Depending on the extent of the cave system, each survey typically involved 2 to 4 surveyors (maximum 4), with a search effort of 2 to 36 person-hours per cave visit. For vertebrates (other than mammals), an effort was made to capture each individual observed either by hand or with dip nets to confirm identification and obtain voucher photographs. A concerted effort was made to capture representatives of each invertebrate species observed as voucher specimens. Specimens were collected by hand with the aid of brushes, aspirator, or fine-meshed dip nets for aquatic taxa, and placed into 70-100\% ethanol. In some instances, Berlese funnels were used to collect tiny specimens within leaves and detritus. Specimens were sorted in the laboratory and identified to the lowest taxonomic level possible using published taxonomic keys and species descriptions. Specimens of several taxonomic groups were sent to experts for identification. In some cases, identification to the species level was not possible due to collection of immature specimens or lack of taxonomic expertise for some groups.

We searched for additional distributional records of invertebrates and vertebrates in North Carolina caves in the scientific literature, unpublished reports, biodiversity databases, and museum accession records. Literature sources included peer-reviewed journals, books, proceedings, theses and dissertations, government reports, and caving organization newsletters. Searches of literature sources included keyword queries of ISI Web of Science, Google Scholar, and Zoological Record. We also queried the VertNet database (http://www.vertnet.org), a web portal to search accessions of over 170 vertebrate museum collections from 12 countries. The U.S. Geological Survey Bat Population Database (Ellison et al., 2003) was queried for bat observations from caves in North Carolina. We also queried the 
Symbiota Collections of Arthropods Network (SCAN) database (https://scan-bugs.org/portal/), a web portal to search over 21 million arthropod records in over 215 museum collections, and InvertEBase (http://www.invertebase.org/portal/index.php), a web portal to search 18 arthropod and mollusk collections in the United States. Institutions for which accessions included specimens collected from North Carolina include Carnegie Museum of Natural History (CMNH), Denver Museum of Nature \& Science (DMNS), Field Museum of Natural History (FMNH), North Carolina Museum of Natural Sciences (NCSM), University of Florida Museum of Natural History (UF), Museum of Comparative Zoology at Harvard University, Museum of Vertebrate Zoology at University of California-Berkeley (MVZ), Royal Ontario Museum (ROM), San Diego State University Museum of Biodiversity (SDSU), Smithsonian National Museum of Natural History (USNM), and University of Michigan Museum of Zoology (UMMZ). We also searched the North Carolina Natural Heritage Program database for occurrences (https://www.ncnhp.org/data/species-community-search).

The annotated list of invertebrate and vertebrate fauna includes the scientific name, authority, ecological classification, common name, and conservation status for each species. Taxonomic nomenclature primarily followed the Encyclopedia of Life (http://www.eol.org). We used common names from published sources when available (e.g. NatureServe). We note the type locality for species described from caves or other subterranean habitats in North Carolina. Classifications of cave-associated organisms (cavernicoles) have been proposed by several authors (e.g., Barr, 1968; Sket, 2008; Culver and Pipan, 2009). We used terminology from Barr (1968) with clarification from Sket (2008) and Culver and Pipan (2009), depending on the taxa, to indicate species found in terrestrial (troglo-) versus aquatic (stygo-) habitats. The four primary ecological categories, with the abbreviations used in the fauna list below and Table 2, were troglobite or stygobite (TB or SB, respectively), troglophile or stygophile (TP or SP) (synonym: eutroglophile), trogloxene or stygoxene (TX or SX) (synonym: subtroglophile), and accidental (AC) (synonym: trogloxene, sensu Sket, 2008). Troglobites and stygobites are obligate cavernicoles with morphological, physiological, and behavioral adaptations for living in subterranean habitats and that have few to no records from surface habitats. Troglophiles and stygophiles frequent subterranean habitats and can complete their life cycles within caves but also may occur in surface habitats. Trogloxenes and stygoxenes use subterranean habitats seasonally, or for only a portion of their life cycles, but also rely significantly on surface habitats. Accidentals are species found in caves only by accident, such as by falling into a pit or being washed into a cave during a flood. We also include species that are intentionally introduced into caves by humans and would not otherwise occur there naturally in this category (e.g., trout). We also denote edaphobites (ED), species thought to be deep-soil inhabitants that may occasionally occur in caves.

The conservation status of each species, based on the International Union for Conservation of Nature (IUCN) Red List of Threatened Species (http://www.iucnredlist.org/; accessed 1 December 2019) and NatureServe (http://www. natureserve.org/; accessed 1 December 2019), is included to provide a better understanding of the distribution and biogeography of subterranean organisms in North Carolina, and to aid in the future conservation and management of this unique fauna. The status of a species according to the U.S. list of threatened and endangered species under the Endangered Species Act is included (http://www.fws.gov/endangered), as well as if a species is included on the list of rare animal species in North Carolina (LeGrand et al., 2014). In addition, we also note species designated "Species of Greatest Conservation Need" in the 2015 North Carolina Wildlife Action Plan (North Carolina Wildlife Resources Commission, 2015).

Seven IUCN Red List categories are recognized on a continuum of increasing extinction risk (International Union for the Conservation of Nature, 2012): Least Concern, Near Threatened, Vulnerable, Endangered, Critically Endangered, Extinct in the Wild, and Extinct. Two additional categories are also recognized: Data Deficient, in which a species has been evaluated, but insufficient data are available to determine a conservation rank; and Not Evaluated, in which a species has yet to be evaluated. Critically Endangered, Endangered, and Vulnerable are considered Threatened categories. Species are classified as Threatened provided they meet one of five criteria (International Union for the Conservation of Nature, 2012): (A) past, present, or projected reduction in population size over three generations; (B) small geographic range in combination with fragmentation, population decline or fluctuations; (C) small population size in combination with decline or fluctuations; (D) very small population or very restricted distribution; or (E) a quantitative analysis of extinction risk. The IUCN Red List classification and associated criteria and subcriteria are presented, if applicable. Subcriteria are detailed in International Union for the Conservation of Nature (2012). NatureServe conservation status ranks are based on a one to five scale, from most to least at risk of extinction (Faber-Langendoen et al., 2012): G1 (Critically Imperiled), G2 (Imperiled), G3 (Vulnerable), G4 (Apparently Secure), and G5 (Secure). Two additional ranks associated with extinction exist: GH (Possibly Extinct) and GX (Presumed Extinct). Finally, species that have not been assessed are assigned GNR (Unranked). A Questionable rank qualifier (?) can be used to denote uncertainty in the conservation status rank (e.g., G2?). Status ranks are assessed at three geographic scales: global (G1-5), national (N1-5), and state (S1-5). Ranks at the global and state scales are given in the text and Table 2. 


\title{
ANNOTATED LIST OF INVERTEBRATE AND VERTEBRATE SUBTERRANEAN FAUNA OF NORTH CAROLINA
}

\author{
Phylum Platyhelminthes \\ Class Turbellaria \\ Order Tricladida \\ Family Planariidae \\ Genus Phagocata
}

\section{Phagocata carolinensis Kenk, 1979 (SB) Carolina Cave Planarian}

LoCALITIES: Burke Co.: One Bat Cave (type locality) ${ }^{1}$; McDowell Co.: Wind Cave ${ }^{2}$.

Conservation Status: IUCN: Not assessed; NatureServe: G1G2 (S1S2 in North Carolina).

COMmENTS: This cave flatworm is known only from these two caves.

References: ${ }^{1}$ Kenk (1979b); ${ }^{2}$ Kenk (1987).

\author{
Phagocata holleri Kenk, 1979 (SB) Holler's Cave Planarian \\ LoCALITIES: Ashe Co.: Mount Jefferson Cave (type locality). \\ Conservation Status: IUCN: Not assessed; NatureServe: G1G2 (S1S2 in North Carolina). \\ COMmENTS: This cave flatworm is known only from the type locality. Specimens have been collected from a seep within \\ the cave. This species was named in honor of the lead author who first discovered this stygobite. \\ REFERENCES: ${ }^{1}$ Kenk (1979a).
}

Phagocata morgani (Stevens \& Boring, 1906) (SP) Morgan's Planarian

LocAlities: Avery Co.: Frying Pan Cave*; McDowell Co.: Staircase Cave*; Rutherford Co.: Bat Cave complex ${ }^{1}$; Wilkes Co.: Devils Den Cave*.

Conservation Status: IUCN: Not assessed; NatureServe: G4G5 (SNR in North Carolina).

COMments: This species is known from spring-runs, springs, and caves in eastern North America.

ReferenCES: ${ }^{1}$ Hertl (1981); ${ }^{*}$ this study.

\section{Phagocata procera Kenk, 1984 (SB) A Cave Planarian}

LOCALITIES: Burke Co.: Fifty-Fifty Fissure ${ }^{2}$, Flatworm Fissure2; Jackson Co.: Cat Den Cave (type locality) ${ }^{1,2}$; McDowell Co.: Lake Tahoma Cave'; Mitchell Co.: Buckshot Cave²; Yancey Co.: seep on State Road 128², Mount Mitchell².

Conservation Status: IUCN: Not assessed; NatureServe: G1G2 (S1S2 in North Carolina).

COMmENTS: This stygobite was described from Cat Den Cave in Jackson County but has been found in several caves and springs in western North Carolina.

RefERENCES: ${ }^{1}$ Kenk (1984); ${ }^{2}$ Kenk (1987).

\section{Phagocata pygmaea Kenk, 1987 (SB) A Cave Planarian}

LOCALITIES: Stokes Co.: Turtle Shell Cave (type locality) ${ }^{1}$.

Conservation Status: IUCN: Not assessed; NatureServe: GNR (SNR in North Carolina).

COMMENTS: This small cave flatworm is known only from the type locality.

REFERENCEs: ${ }^{1}$ Kenk (1987).

\section{Phagocata spuria Kenk, 1987 (SB) A Cave Planarian}

LOCALITIES: McDowell Co.: Bennett's Mill Cave (type locality)1.

Conservation StATUs: IUCN: Not assessed; NatureServe: GNR (SNR in North Carolina).

COMMENTS: This species is similar in appearance to $P$. morgani but is known only from the type locality.

References: ${ }^{1}$ Kenk (1987).

Phagocata sp. (SP) A Planarian

LocAlities: Ashe Co.: Mount Jefferson Cave*; Burke Co.: Crystal Worm Cave*; Flaky Lips Fissure Cave*; Henderson Co.: Stillers Cave*.

COMmENTS: Flatworms collected from these caves were immature and could not be identified confidently to species.

REFERENCES: * this study.

\section{Phagocata sp.? (SP) A Planarian}

LocAlitIES: Avery Co.: Charlie's Ridge Bat Cave*; Buncombe Co.: Cedar Cliff Cave*; Mitchell Co.: Buckshot Cave*; Surry Co.: Mitchell River Cave*. 
COMmENTS: These immature flatworms resembled Phagocata but may be another related genus.

REFERENCES: * this study.

\author{
Phylum Annelida \\ Class Clitellata \\ Order Haplotaxida \\ Family Acanthodrilidae \\ Genus Diplocardia
}

\title{
Diplocardia caroliniana Eisen, 1899 (ED) An Earthworm
}

LoCALITIES: Macon Co.: Marie Cave1.

Conservation Status: IUCN: Not assessed; NatureServe: GNR (SNR in North Carolina).

Comments: Diplocardia sp. have been reported from caves in Illinois and Missouri (Peck and Lewis, 1978).

RefERENCES: ${ }^{1}$ Reynolds (1994).

\section{Family Lumbricidae \\ Genus Aporrectodea}

\section{Aporrectodea trapezoides (Duges, 1828) (ED) Southern Worm}

LOCALITIES: Macon Co.: Horse Cave ${ }^{1}$, Marie Cave'1.

Conservation Status: IUCN: Not assessed; NatureServe: GNR (SNR in North Carolina).

Comments: This species has also been reported from caves in Georgia, Illinois, and Missouri (Peck and Lewis, 1978;

Reeves and Reynolds, 1999; Reeves et al., 2000).

REFERENCEs: ${ }^{1}$ Reynolds (1994).

\section{Genus Dendrobaena}

\section{Dendrobaena octaedra (Savigny, 1826) (ED) Octagonal-tail Worm}

LocAlitIES: Macon Co.: Horse Cave ${ }^{1}$, Marie Cave1.

Conservation Status: IUCN: Not assessed; NatureServe: GNR (SNR in North Carolina).

COMMENTS: This species has also been reported from a cave in Georgia (Reeves and Reynolds, 1999).

REFERENCES: ${ }^{1}$ Reynolds (1994).

\section{Genus Dendrodrilus}

\section{Dendrodrilus rubidus (Savigny, 1826) (ED) European Earthworm}

LocAlities: Macon Co.: Horse Cave1.

Conservation Status: IUCN: Not assessed; NatureServe: GNR (SNR in North Carolina).

Comments: This species has also been reported from several caves in Georgia, Illinois, Missouri, Tennessee and New Brunswick, Canada (McAlpine and Reynolds, 1977; Peck and Lewis, 1978; Reeves and Reynolds, 1999; Reeves et al., 2000).

REFERENCES: ${ }^{1}$ Reynolds (1994).

\section{Genus Eisenoides}

Eisenoides lonnbergi (Michaelsen, 1894) (ED) American Grey Soil Worm

Localities: Macon Co.: Horse Cave1; Rockingham Co.: Pine Hill Cave1.

Conservation Status: IUCN: Not assessed; NatureServe: GNR (SNR in North Carolina).

COMmENTS: The only reported cave records of this wide-ranging, common earthworm are from North Carolina to date.

REFERENCES: ${ }^{1}$ Reynolds (1994).

\section{Genus Lumbricus}

\section{Lumbricus rubellus Hoffmeister, 1843 (ED) Red Marshworm}

Localities: Macon Co.: Horse Cave'.

Conservation Status: IUCN: Not assessed; NatureServe: GNR (SNR in North Carolina).

Comments: This species has also been reported from caves in Georgia and Illinois (Peck and Lewis 1978; Reeves et al. 2000).

References: ${ }^{1}$ Reynolds (1994). 


\section{Genus Octolasion}

Octolasion tyrtaeum (Savigny, 1826) (ED) Woodland White Worm Localities: Macon Co.: Horse Cave'; Rockingham Co.: Pine Hill Cave1.

Conservation Status: IUCN: Not assessed; NatureServe: GNR (SNR in North Carolina).

Comments: This species has also been reported from caves in Georgia, Illinois, and Tennessee (Holsinger and Peck, 1971; Peck and Lewis, 1978; Lewis, 2005a), including Great Smoky Mountains National Park (Reeves, 2000b).

REFERENCEs: ${ }^{1}$ Reynolds (1994).

\section{Family Sparganophilidae \\ Genus Sparganophilus}

\section{Sparganophilus eiseni (Smith, 1895) (ED) American Mudworm}

Localities: Macon Co.: Horse Cave'.

Conservation Status: IUCN: Not assessed; NatureServe: GNR (SNR in North Carolina).

COMmENTS: This species is known from several records in the eastern United States and southeastern Canada, including North Carolina (Reynolds, 1980; McAlpine et al., 2001).

REFERENCES: ${ }^{1}$ Reynolds (1994).

\section{Phylum Mollusca \\ Class Gastropoda \\ Order Neotaenioglossa \\ Family Pleuroceridae \\ Genus Elimia}

Elimia proxima (Say, 1825) (SX) Sprite Elimia

LoCALITIES: Rutherford Co.: McGrath Fissure*, Rumbling Bald Spring Cave*, Spring Fissure1.

Conservation Status: IUCN: Least Concern; NatureServe: G5 (S5 in North Carolina).

COMmEnTS: This freshwater snail also has been reported from Santee Cave in Orangeburg Co., South Carolina (Reeves, 2001).

References: ${ }^{1}$ Christ (2003); * this study.

\section{Order Stylommatophora \\ Family Gastrodontidae Genus Zonitoides}

Zonitoides elliotti (Redfield, 1856) (TX) Green Gloss

LoCALITIES: Macon Co.: Horse Cave'; McDowell Co.: Staircase Cave².

Conservation Status: IUCN: Not assessed; NatureServe: G4 (S4 in North Carolina).

REFERENCES: ${ }^{1}$ Invertebase: CMNH; ${ }^{2}$ Invertebase: UF.

\section{Family Haplotrematidae Genus Haplotrema}

\section{Haplotrema concavum (Say, 1821) (TX/AC) Grayfoot Lancetooth Snail}

LocalitIES: Davidson Co.: Boone's Cave'.

Conservation Status: IUCN: Not assessed; NatureServe: G5 (S5 in North Carolina).

REFERENCES: ${ }^{1}$ Invertebase: FMNH.

\section{Haplotrema sp. (TX/AC) A Lancetooth Snail}

LoCALITIES: Swain Co.: Blowing Springs Cave*.

COMmENTS: A carnivorous genus that feeds on other gastropod.Two species have been documented from western North Carolina, H. concavum and the rarer H. kendeighi (Dourson, 2013).

REFERENCES: * this study.

\section{Family Helicodiscidae \\ Genus Helicodiscus}

Helicodiscus saludensis (Morrison, 1937) (TX/AC) Corncob Snail

LOCALITIES: Rutherford Co.: Ice Cave ${ }^{1}$.

Conservation Status: IUCN: Not assessed; NatureServe: G1 (S1? in North Carolina).

REFERENCES: ${ }^{1}$ Invertebase: UF. 


\section{Family Polygyridae \\ Genus Inflectarius}

Inflectarius inflectus (Say, 1821) (TX) Shagreen

LocAlitIES: Davidson Co.: Boone's Cave'; Henderson Co.: Hog Rock Cave*, Middle Fork Shelter Cave no. 2*; Rutherford Co.: McGrath Fissure*, Rumbling Bald Spring Cave*, Spring Fissure ${ }^{2}$.

Conservation Status: IUCN: Not assessed; NatureServe: G5 (S5 in North Carolina).

ReferenCes: ${ }^{1}$ Invertebase: FMNH; ${ }^{2}$ Christ (2003), ${ }^{*}$ this study.

\section{Genus Mesodon}

\section{Mesodon andrewsae Binney, 1879 (AC) Balsam Globe}

Localities: Polk Co.: World's Edge Fissure*.

Conservation Status: IUCN: Not assessed; NatureServe: G3 (S2S3 in North Carolina); Listed as a Species of Greatest Conservation Need in North Carolina (North Carolina Wildlife Resources Commission, 2015).

REFERENCES: * this study.

\section{Mesodon thyroidus (Say, 1816) (AC) Whitelip Globe}

LoCALITIES: Rutherford Co.: Devil's Smokehouse Cave'.

Conservation Status: IUCN: Not assessed; NatureServe: G5 (S5 in North Carolina).

REFERENCES: 1 Invertebase: NCSM.

\section{Mesodon sp. (AC) A Globe}

LocAlitIES: Madison Co.: Anthodite Cave'.

Comments: This occurrence may be $M$. andrewsae, $M$. thyroidus, or another species.

REFERENCES: ${ }^{1}$ Invertebase: UF.

\section{Genus Patera}

\section{Patera perigrapta (Pilsbry, 1894) (TX) Engraved Bladetooth}

Localities: Henderson Co.: Hog Rock Cave*; Madison Co.: French Broad Cave'; Shutin Creek Cave1, Polk Co.: Beast Cave $^{1}$, Rutherford Co.: Bat Cave ${ }^{2}$, Rumbling Bald Cave ${ }^{1}$, White Root Shelter*.

Conservation Status: IUCN: Not assessed; NatureServe: G5 (S5? in North Carolina).

RefERENCES: ${ }^{1}$ Invertebase: UF; ${ }^{2}$ Invertebase: NCSM; * this study.

\section{Family Zonitidae \\ Genus Glyphyalinia}

\section{Glyphyalinia carolinensis (Cockerell, 1890) (TX/AC) Spiral Mountain Glyph}

LocAlities: McDowell Co.: Staircase Cave'.

Conservation Status: IUCN: Not assessed; NatureServe: G4 (S4 in North Carolina).

REFERENCES: ${ }^{1}$ Invertebase: UF.

\section{Glyphyalinia sp. (TX/AC) A Glyph}

LOCALITIES: Rutherford Co.: Western Talus Tunnel*.

References: * this study.

\section{Genus Mesomphix}

\section{Mesomphix andrewsae (Pilsbry, 1895) (TX/AC) Mountain Button}

LocAlitIES: Swain Co.: Lost Nantahala Cave1.

Conservation Status: IUCN: Not assessed; NatureServe: G3G4 (S3S4 in North Carolina).

REFERENCES: ${ }^{1}$ Invertebase: UF.

\section{Mesomphix latior (Pilsbry, 1900) (TX/AC) Broad Button}

LoCALITIES: Swain Co.: Lost Nantahala Cave1.

Conservation Status: IUCN: Not assessed; NatureServe: G3G4 (S2S3 in North Carolina); Listed as a Species of Greatest Conservation Need in North Carolina (North Carolina Wildlife Resources Commission, 2015).

REFERENCES: ${ }^{1}$ Invertebase: UF. 


\title{
Mesomphix subplanus (Binney, 1842) (TX/AC) Flat Button
}

LoCALITIES: Rutherford Co.: Bat Cave'.

Conservation Status: IUCN: Not assessed; NatureServe: G3G4 (S3S4 in North Carolina).

REFERENCES: ${ }^{1}$ Invertebase: NCSM.

\author{
Phylum Arthropoda \\ Subphylum Chelicerata \\ Class Arachnida \\ Order Araneae \\ Family Agelenidae \\ Genus Agelenopsis
}

Agelenopsis sp. (TX/AC) A Grass Spider

LoCALITIES: Polk Co.: Little Warrior Mountain Cave'; Rutherford Co.: Rumbling Bald Cave1.

RefERENCES: ${ }^{1}$ Gaddy (1986b).

\section{Genus Coras}

\section{Coras sp. (TX) A Funnel Weaver Spider}

Localities: Henderson Co.: Hog Rock Cave2; Polk Co.: Little Warrior Mountain Cave1; Rutherford Co.: Bat Cave1, Cane Creek Mountain Cave ${ }^{2}$, Rumbling Bald Cave ${ }^{1}$, Sliding Rock Cave*, Western Talus Tunnel²; Yancey Co.: Celo Cave*.

References: ${ }^{1}$ Gaddy (1986b); ${ }^{2}$ Christ (2003); * this study.

\section{Genus Wadotes}

\section{Wadotes bimucronatus (Simon, 1898) (TX/AC) Hackledmesh Weaver Spider}

LoCALITIES: Swain Co.: Blowing Springs Cave*

Conservation Status: IUCN: Not assessed; NatureServe: Not assessed.

REFERENCES: * this study.

Wadotes sp. (TX/AC) A Weaver Spider

LOCALITIES: Rutherford Co.: Bat Cave complex'.

REFERENCES: ${ }^{1}$ Hertl (1981).

\section{Undetermined Genus}

Undetermined species (TX/AC) A Weaver Spider

Localities: Henderson Co.: Devils Kitchen Cave ${ }^{1}$; Rutherford Co.: A-Frame Fissure ${ }^{1}$, Moonshiners Cave*, Rumbling Bald Cave'.

References: ${ }^{1}$ Christ (2003); * this study.

\section{Family Antrodiaetidae \\ Genus Antrodiaetus}

\section{Antrodiaetus unicolor (Hentz, 1842) (TX) Collar Door Spider}

Localities: McDowell Co.: Wind Cave*; Rutherford Co.: Rumbling Bald Cave*, Western Talus Tunnel'.

Conservation StATUS: IUCN: Not assessed; NatureServe: GNR (SNR in North Carolina).

COMMENTS: This is a burrowing species; most records were of wandering adult males.

References: ${ }^{1}$ Christ (2003); ${ }^{*}$ this study.

\section{Family Araneidae \\ Genus Mangora}

Mangora maculata (Keyserling, 1865) (TX/AC) Green-legged Orbweaver

LOCALITIES: Rutherford Co.: Western Talus Tunnel'1.

Conservation Status: IUCN: Not assessed; NatureServe: GNR (SNR in North Carolina).

References: ${ }^{1}$ Christ (2003).

\section{Family Cybaeidae}

Genus Calymmaria

Calymmaria persica (Hentz, 1847) (TX) A Dwarf Sheet Spider

LocAlities: Caldwell Co.: Nats Cave*; Rutherford Co.: Bat Cave*. 
Conservation Status: IUCN: Not assessed; NatureServe: GNR (SNR in North Carolina).

COMmEnTs: This is one of over 30 known species of Calymmaria, all from North America.

REFERENCES: * this study.

\section{Family Hypochilidae \\ Genus Hypochilus}

\section{Hypochilus coylei Platnick, 1987 (TX) A Lampshade Weaver}

Localities: Polk Co.: Little Warrior Mountain Cave²; Rutherford Co.: Bat Cave², Breakdown Cave', Rumbling Bald Cave², Sliding Rock Cave*.

Conservation Status: IUCN: Not assessed; NatureServe: G3? (S3? in North Carolina).

COMMENTS: This species lives an average of two years and feeds on other spiders and crickets.

RefERENCES: ${ }^{1}$ SCAN: SDSU; ${ }^{2}$ Gaddy $(1986 \mathrm{~b}) ;{ }^{*}$ this study.

Hypochilus pococki Platnick, 1987 (TX) Pocock's Lampshade-web Spider

LoCAlitIES: Avery Co.: Elk River Cave²; McDowell Co.: Linville Caverns"; Transylvania Co.: Snake Den no. 2 Cave*.

Conservation Status: IUCN: Not assessed; NatureServe: G4G5 (SNR in North Carolina).

REFERENCES: ${ }^{1}$ SCAN: DMNS; ${ }^{2}$ SCAN: SDSU; * this study.

Hypochilus sp. (TX) A Lampshade Weaver

Localities: Henderson Co.: Devils Kitchen Cave'; Madison Co.: French Broad Cave*; Polk Co.: Garnet Shelter Cave ${ }^{1}$, World's Edge Cave ${ }^{1}$; Rutherford Co.: Breakdown Cave* ${ }^{*}$ Cane Creek Mountain Cave ${ }^{1}$, Moonshiners Cave*, Western Talus Tunnel'.

COMMENTS: The records represent collections of immatures or visual observations only that could not be identified to species.

RefERENCES: ${ }^{1}$ Christ (2003); * this study.

\section{Family Linyphiidae \\ Genus Microneta}

\section{Microneta viaria (Blackwell, 1841) (TX/AC) Small Sheetweb Weaver}

Localities: Polk Co.: World's Edge Fissure'.

Conservation Status: IUCN: Not assessed; NatureServe: Not assessed.

RefERENCES: ${ }^{1}$ Christ (2003).

\section{Genus Origanates}

Origanates rostratus (Emerton, 1882) (TX/AC) A Sheetweb Weaver

LOCALITIES: Henderson Co.: Stillers Cave*.

Conservation status: IUCN: Not assessed; NatureServe: GNR (SNR in North Carolina).

REFERENCES: * this study.

Genus Phanetta

Phanetta subterranea (Emerton, 1875) (TB) Subterranean Sheetweb Spider

LocAlitIES: McDowell Co.: Lake Tahoma Cave*; Rutherford Co.: Breakdown Cave*, White Root Shelter'; Yancey Co.: Cooper's Cave*.

Conservation Status: IUCN: Not assessed; NatureServe: G5 (SNR in North Carolina).

Comments: Like many linyphiids, this species is likely more common in North Carolina caves than records indicate but easily overlooked due to its small size.

References: ${ }^{1}$ Christ (2003); ${ }^{*}$ this study.

\section{Genus Porrhomma}

\section{Porrhomma cavernicola (Keyserling, 1886) (TB) Appalachian Cave Spider}

LoCALITIES: Swain Co.: Blowing Springs Cave*, Lost Nantahala Cave*.

Conservation StATUS: IUCN: Not assessed; NatureServe: G5 (SNR in North Carolina).

COMMENTS: This wide-ranging troglobite is known from several caves in the Appalachian Valley and Ridge and Interior Low Plateau (Fong et al., 2012; Niemiller et al., 2013).

REFERENCES: * this study. 


\section{Undetermined Genus}

Undetermined species (TX/AC) A Sheetweb Spider

LoCALITIES: Swain Co.: Blowing Springs Cave*.

COMMENTS: The specimen was immature.

REFERENCES: * this study.

\section{Family Nesticidae \\ Genus Nesticus}

\section{Nesticus brimleyi Gertsch, 1984 (TB) A Cave Cobweb Spider}

LoCAlities: Polk Co.: Little Warrior Mountain Cave ${ }^{1,4,5, * ;}$ Rutherford Co.: A-Frame Fissure ${ }^{7}$, Bat Cave complex ${ }^{2,3,5}$, Breakdown Cave ${ }^{3,6}$, Ice Cave $^{3}$, Moonshiners Cave ${ }^{*}$ Rumbling Bald Cave (type locality) ${ }^{3,5}$, Rumbling Bald Mountain Cave ${ }^{7}$, Rumbling Bald Spring Cave*, Sliding Rock Cave ${ }^{7}$.

Conservation status: IUCN: Not assessed; NatureServe: G1G2 (S1? in North Carolina).

COMMENTS: This troglobite is endemic to North Carolina.

References: ${ }^{1}$ NCNHP; ${ }^{2}$ Hertl (1981); ${ }^{3}$ Gertsch (1984); ${ }^{4,5}$ Gaddy (1986a,b); ${ }^{6}$ Hedin (1997b); ${ }^{7}$ Christ (2003); ${ }^{*}$ this study.

\section{Nesticus carolinensis Gertsch, 1984 (TP) Linville Caverns Spider}

Localities: McDowell Co.: Lime Kiln Cave*, Linville Caverns (type locality) ${ }^{1-4}$, Staircase Cave*.

Conservation status: IUCN: Not assessed; NatureServe: G1? (S1 in North Carolina).

Comments: Gertsch (1984) considered this species a troglophile despite being known only from caves in McDowell County.

RefERENCES: ${ }^{1}$ NCNHP; 2 Gertsch (1984); ${ }^{3,4}$ Hedin (1997a,b); * this study.

\section{Nesticus cooperi Gertsch, 1984 (TP) Lost Nantahala Cave Spider}

LoCALITIES: Swain Co.: Blowing Springs Cave ${ }^{1-3}$, Lost Nantahala Cave (type locality) ${ }^{2,3}$.

Conservation status: IUCN: Not assessed; NatureServe: G1 (S1 in North Carolina).

COMMENTS: Although described from a cave, this species is also known from surface records.

RefERENCES: ${ }^{1}$ NCNHP; ${ }^{2}$ Gertsch (1984); ${ }^{3}$ Hedin (1997b).

Nesticus mimus Gertsch, 1984 (TP) A Cobweb Spider

LOCALITIES: Caldwell Co.: Burnt Field Branch Cave*.

Conservation status: IUCN: Not assessed; NatureServe: G2 (S2? in North Carolina).

Comments: This species also is known from surface collections in southwestern Virginia and northwestern North Carolina (Gertsch, 1984).

REFERENCES: * this study.

\section{Nesticus sp. (TP) A Cobweb Spider}

Localities: Madison Co.: Mine Hollow Cave*; McDowell Co.: Wind Cave*; Yancey Co.: Celo Cave*.

COMmENTS: Immatures from these caves could not be identified to species.

REFERENCES: * this study.

\section{Family Pisauridae Genus Dolomedes}

\section{Dolomedes sp. (TX) A Fishing Spider}

LocAlitIES: Avery Co.: Charlie's Ridge Bat Cave*.

COMmENTS: Fishing spiders are observed not infrequently around the entrances and twilight zones of caves. This individual was observed crawling along the floor of a pool pursuing an undescribed species of Stygobromus amphipod.

REFERENCES: * this study.

\section{Family Tetragnathidae \\ Genus Meta}

\section{Meta ovalis (Gertsch, 1933) (TP) Cave Orbweaver}

LoCALITIES: Avery Co.: Cranberry Mines*; Jackson Co.: Kitchen Cave*; McDowell Co.: Lime Kiln Cave*, Linville Caverns*, Linville Indian Cave ${ }^{*}$, Pseudo-Saltpetre Cave*, Staircase Cave*, Wind Cave*; Polk Co.: Little Warrior Mountain Cave ${ }^{1-3}$; Rutherford Co.: Bat Cave complex ${ }^{1,3}$, Breakdown Cave*, Ice Cave*, Moonshiners Cave*, Rumbling Bald Cave ${ }^{3}$, Rumbling Bald Spring Cave*, Sliding Rock Cave*; Swain Co.: Blowing Springs Cave*, Lost Nantahala Cave*; Transylvania Co.: Snake Den no. 2 Cave*; Yancey Co.: Celo Cave*, Cooper's Cave*. 
Conservation status: IUCN: Not assessed; NatureServe: GNR (SNR in North Carolina).

Comments: This species is widely distributed in caves in North Carolina and throughout its range. It is also commonly reported from culverts, basements, and other dark recesses.

ReferenCEs: ${ }^{1}$ Hertl (1981); ${ }^{2,3}$ Gaddy (1986a,b); ${ }^{*}$ this study.

\section{Family Theridiidae \\ Genus Cryptachaea}

Cryptachaea rupicola (Emerton, 1882) (TX) A Cobweb Spider

LOCALITIES: Rutherford Co.: Breakdown Cave*, Rumbling Bald Cave*.

Conservation status: IUCN: Not assessed; NatureServe: GNR (SNR in North Carolina).

REFERENCES: * this study.

\section{Genus Parasteatoda}

\section{Parasteatoda tepidariorum (Koch, 1841) (TX) Common House Spider}

Localities: Madison Co.: Anthodite Cave*, French Broad Cave*, Shutin Creek Cave*; McDowell Co.: Linville Indian Cave*.

Conservation StATUS: IUCN: Not assessed; NatureServe: GNR (SNR in North Carolina).

REFERENCES: * this study.

\section{Genus Tidarren}

Tidarren sisyphoides (Walckenaer, 1841) (TX/AC) Tent Cobweb Spider

LoCALITIES: Polk Co.: Little Warrior Mountain Cave1,2; Rutherford Co.: Bat Cave ${ }^{2}$, Rumbling Bald Cave2.

Conservation Status: IUCN: Not assessed; NatureServe: Not assessed.

RefERENCES: ${ }^{1,2}$ Gaddy (1986a,b).

\section{Undetermined Genus}

Undetermined species (TXIAC) A Cobweb Spider

LoCALITIES: Henderson Co.: Devils Kitchen Cave'.

References: ${ }^{1}$ Christ (2003).

\section{Family Theridiosomatidae Genus Theridiosoma}

Theridiosoma gemmosum (Koch, 1877) (TX) A Ray Orbweaver

LocAlities: Caldwell Co.: Nats Cave*; McDowell Co.: Staircase Cave*.

Conservation status: IUCN: Not assessed; NatureServe: GNR (SNR in North Carolina).

REFERENCES: * this study.

\section{Order Opiliones \\ Family Phalangiidae \\ Genus Leiobunum}

\section{Leiobunum bicolor (Wood, 1870) (TX) A Harvestman}

Localities: McDowell Co.: Lime Kiln Cave*, Linville Caverns*, Staircase Cave*; Rutherford Co.: McGrath Fissure1.

Conservation Status: IUCN: Not assessed; NatureServe: Not assessed.

COMMENTS: This genus is known to overwinter, often in large aggregations, in caves.

RefERENCES: ${ }^{1}$ Christ (2003); * this study.

\section{Leiobunum sp. (TX) A Harvestman}

LoCALITIES: Avery Co.: Cranberry Mines*; Swain Co.: Lost Nantahala Cave*; Transylvania Co.: Snake Den no. 2 Cave*; Yancey Co.: Celo Cave*, Isom Mica Mine*.

COMMENTS: These records may be $L$. bicolor.

REFERENCES: * this study. 


\section{Family Phalangodidae \\ Genus Bishopella}

Bishopella laciniosa (Crosby \& Bishop, 1924) (TP) Bishop's Harvestman

LOCALITIES: McDowell Co.: Linville Caverns ${ }^{1}$.

Conservation Status: IUCN: Not assessed; NatureServe: Not assessed.

COMMENTS:

REFERENCES: ${ }^{1}$ SCAN: SDSU.

\section{Bishopella sp. (TP) A Harvestman}

LoCALITIES: Swain Co.: Blowing Springs Cave*, Lost Nantahala Cave*.

COMMENTS: These records are probably $B$. laciniosa.

REFERENCES: * this study.

\section{Family Travuniidae \\ Genus Theromaster}

Theromaster brunneus (Banks, 1902) (TX) A Harvestman

LocAlities: Henderson Co.: Devils Kitchen Cave ${ }^{3}$; McDowell Co.: Linville Caverns ${ }^{1}$; Polk Co.: Garnet Shelter Cave ${ }^{3}$;

Rutherford Co.: Bat Cave complex ${ }^{1,2}$.

ConserVATION STATUS: IUCN: Not assessed; NatureServe: Not assessed.

COMMENTS: This armored harvestman is collected occasionally from caves.

RefERENCES: ${ }^{1}$ SCAN: SDSU; ${ }^{2}$ Hertl (1981); ${ }^{3}$ Christ (2003).

\section{Order Pseudoscorpiones \\ Family Chthoniidae \\ Genus Kleptochthonius}

Kleptochthonius sp. (TP/TX) A Pseudoscorpion

LocAliTIES: Henderson Co.: Devil's Kitchen Cave*.

REFERENCES: * this study.

\section{Undetermined Genus}

Undetermined species (TX/AC) A Pseudoscorpion

LOCALITIES: Swain Co.: Blowing Springs Cave*.

REFERENCES: * this study.

\section{Family Neobisiidae \\ Genus Microcreagris}

Microcreagris sp. (TP/TX) A Pseudoscorpion

LOCALITIES: Polk Co.: Zig-Zag Fissure*.

REFERENCES: * this study.

\section{Subclass Acari \\ Order Mesostigmata \\ Family Arctacaridae \\ Undetermined Genus}

Undetermined species (TX/AC) A Mite

LoCALITIES: Rutherford Co.: Bat Cave complex'.

References: ${ }^{1}$ Hertl (1981).

\section{Family Parasitidae \\ Undetermined Genus}

Undetermined species (TX/AC) A Mite

LocAlities: Henderson Co.: Devils Kitchen Cave'; Rutherford Co.: Block Creep Cave'1.

References: ${ }^{1}$ Christ (2003). 


\section{Family Parholaspididae \\ Undetermined Genus}

Undetermined species (TX/AC) A Mite

LOCALITIES: Henderson Co.: Devils Kitchen Cave'1.

References: ${ }^{1}$ Christ (2003).

\section{Order Sarcoptiformes \\ Family Lohmanniidae \\ Genus Lohmannia}

\section{Lohmannia sp. (TX) A Mite}

LOCALITIES: Rutherford Co.: Bat Cave complex'1.

References: ${ }^{1}$ Hertl (1981).

Family Phthiracaridae

Genus Steganacarus

Steganacarus sp. (TX) A Mite

LOCALITIES: Rutherford Co.: Bat Cave complex ${ }^{1}$.

RefERENCES: ${ }^{1}$ Hertl (1981).

\section{Order Trombidiformes \\ Family Bdellidae \\ Undetermined Genus}

Undetermined species (TP/TX) A Mite

LocAlITIES: Rutherford Co.: Block Creep Cave1.

References: ${ }^{1}$ Christ (2003).

\section{Family Rhagidiidae \\ Undetermined Genus}

Undetermined species (TP) A Mite

LoCAlities: Polk Co.: Garnet Shelter Cave ${ }^{1}$, World's Edge Cave'1.

References: ${ }^{1}$ Christ (2003).

\section{Subphylum Hexapoda \\ Class Collembola \\ Order Entomobryomorpha \\ Family Entomobryidae \\ Genus Entomobra}

Entomobrya quadrilineata Bueker, 1939 (AC) A Springtail

LoCALITIES: Rutherford Co.: Block Creep Cave*.

Conservation Status: IUCN: Not assessed; NatureServe: Not assessed.

COMMENTS: This well-pigmented and eyed springtail is occasionally collected in caves.

References: * this study.

\section{Genus Pseudosinella}

Pseudosinella collina Wray, 1952 (TP) A Springtail

LoCALITIES: Polk Co.: Garnet Shelter Cave'1.

Conservation Status: IUCN: Not assessed; NatureServe: GNR (SNR in North Carolina).

COMmENTS: This troglophilic springtail is known from several cave and surface records in the eastern United States.

References: ${ }^{1}$ Christ (2003).

Pseudosinella flatua Christiansen \& Bellinger, 1996 (TB) Blowing Springs Cave Springtail

LoCALITIES: Swain Co.: Blowing Springs Cave (type locality) ${ }^{1,2}$.

Conservation Status: IUCN: Not assessed; NatureServe: G1G2 (S1S2 in North Carolina).

COMMENTS: This troglobite is known only from the type locality.

RefERENCES: ${ }^{1}$ Christiansen and Bellinger (1996); ${ }^{2}$ SCAN: MCZ. 
Pseudosinella gisini carolina Christiansen \& Bellinger, 1996 (TB) Carolina Cave Springtail

LocAlITIES: Rutherford Co.: McGrath Fissure (type locality) $)^{1,2}$, Rumbling Bald Cave2.

Conservation Status: IUCN: Not assessed; NatureServe: G3G4T1T2 (S1S2 in North Carolina).

COMmEnTS: This subspecies is known only from these two caves.

ReferenCes: ${ }^{1}$ Christ (2003); ${ }^{2}$ Christiansen and Bellinger (1996).

Pseudosinella orba Christiansen, 1961 (TB) A Cave Springtail

Localities: Polk Co.: World's Edge Cave'.

Conservation Status: IUCN: Not assessed; NatureServe: G3G4 (SU in North Carolina).

Comments: This species also is known from caves in Kentucky, Tennessee, Virginia, and West Virginia.

REFERENCES: ${ }^{1}$ Christiansen Grinnell Database.

\section{Pseudosinella pecki Christiansen \& Bellinger, 1980 (TB) Peck's Cave Springtail}

Localities: Swain Co.: Hot Pit Cave1.

Conservation Status: IUCN: Not assessed; NatureServe: G2G3 (SNR in North Carolina).

Comments: This troglobite is known from caves in several states in the eastern United States (Christiansen and Bellinger, 1980; Reeves et al., 2000).

ReFERENCES: ${ }^{1}$ Christiansen Grinnell Database.

\section{Pseudosinella vespera Christiansen \& Bellinger, 1996 (TB) Bat Cave Springtail}

LoCALITIES: Rutherford Co.: Bat Cave (type locality) ${ }^{1-4}$; Swain Co.: Blowing Springs Cave ${ }^{3}$.

Conservation Status: IUCN: Not assessed; NatureServe: G2 (S1S2 in North Carolina).

Comments: This troglobite is known from these two North Carolina caves as well as a cave in Bland Co., Virginia.

RefERENCES: ${ }^{1}$ Christiansen Grinnell Database; ${ }^{2}$ Hertl (1981); ${ }^{3}$ Christiansen and Bellinger (1996), ${ }^{4}$ SCAN: MCZ.

\section{Pseudosinella sp. nov.? (TB) A Springtail}

LoCALITIES: Rutherford Co.: White Root Shelter ${ }^{1}$.

COMMENTS: This record may represent a new species or one of the described troglobitic taxa.

RefERENCES: ${ }^{1}$ Christ (2003).

\section{Family Isotomidae \\ Genus Folsomia}

Folsomia candida Willem, 1902 (TP) White Springtail

LOCALITIES: Swain Co.: Blowing Springs Cave*.

Conservation Status: IUCN: Not assessed; NatureServe: GNR (SNR in North Carolina).

REFERENCES: * this study.

\section{Family Lepidocyrtidae \\ Genus Lepidocyrtus}

Lepidocyrtus sp. (TX) A Springtail

LocAlities: Henderson Co.: Devils Kitchen Cave ${ }^{1}$, Hog Rock Cave1; Polk Co.: World's Edge Cave1; Rutherford Co.: Sliding Rock Cave ${ }^{1}$, White Root Shelter ${ }^{1}$.

RefERENCES: ${ }^{1}$ Christ (2003).

\section{Family Tomoceridae \\ Genus Pogonognathellus}

Pogonognathellus bidentatus (Folsom, 1913) (TP) A Springtail

LocALITIES: Caldwell Co.: Burnt Field Branch Cave*; McDowell Co.: Lake Tahoma Cave*; Polk Co.: Little Warrior Mountain Cave*, World's Edge Cave²; Rutherford Co.: Bat Cave complex'; Rimstone Skull Cave ${ }^{2}$, Sliding Rock Cave²; Swain Co.: Blowing Springs Cave*.

Conservation Status: IUCN: Not assessed; NatureServe: Not assessed.

RefERENCES: ${ }^{1}$ Hertl (1981); ${ }^{2}$ Christ (2003); * this study.

Pogonognathellus flavescens (Tullberg, 1871) (TP) A Springtail

LoCALITIES: Polk Co.: World's Edge Cave'; Swain Co.: Blowing Springs Cave*.

Conservation Status: IUCN: Not assessed; NatureServe: Not assessed.

References: ${ }^{1}$ Christ (2003); * this study. 


\section{Pogonognathellus sp. (TP) A Springtail}

LoCALITIES: Rutherford Co.: Middle Bat Cave (Bat Cave complex)*.

REFERENCES: * this study.

\section{Order Poduromorpha \\ Family Onychiuridae \\ Genus Onychiurus}

Onychiurus similis Folsom, 1917 (TP) A Springtail

LOCALITIES: Swain Co.: Blowing Springs Cave*.

Conservation Status: IUCN: Not assessed; NatureServe: Not assessed.

REFERENCES: * this study.

Onychiurus sp. nov.? (TP) A Springtail

LOCALITIES: Henderson Co.: Devils Kitchen Cave'.

COMMENTS: This record may represent an undescribed species.

References: ${ }^{1}$ Christ (2003).

Onychiurus sp. (TP) A Springtail

LocAlities: Henderson Co.: Devils Kitchen Cave ${ }^{1}$.

References: ${ }^{1}$ Christ (2003).

\section{Genus Paronychiurus}

Paronychiurus ramosus (Folsom, 1917) (TP) A Springtail

Localities: Polk Co.: Garnet Shelter Cave ${ }^{1}$, Little Warrior Mountain Cave*.

Conservation Status: IUCN: Not assessed; NatureServe: Not assessed.

References: ${ }^{1}$ Christ (2003); * this study.

\section{Order Symphypleona \\ Family Arrhopalitidae \\ Genus Arrhopalites}

Arrhopalites sp. (TP) A Springtail

LocAlITIES: Swain Co.: Blowing Springs Cave*.

COMMENTS: This specimen was collected from the surface of a cave pool, but unfortunately was lost in the taxonomic lab before species could be determined.

REFERENCES: * this study.

Family Katiannidae

Genus Sminthurinus

\section{Sminthurinus sp. nov.? (TP) A Springtail}

LOCALITIES: Polk Co.: World's Edge Shelter*.

COMMENTS: This record may represent an undescribed species (K. Christensen, pers. comm.).

REFERENCES: * this study.

\section{Class Entognatha \\ Order Diplura \\ Family Campodeidae \\ Genus Litocampa}

\section{Litocampa sp. (TB) A Cave Bristletail}

LoCAlITIES: Henderson Co.: Hog Rock Cave ${ }^{1}$, Stillers Cave*; Madison Co.: Mine Hollow Cave*, Shut-In Creek Cave*; McDowell Co.: Lime Kiln Cave*, Staircase Cave*, Wind Cave*; Polk Co.: Little Warrior Mountain Cave*, Zig-Zag Fissure"; Rutherford Co.: McGrath Fissure ${ }^{1}$; Swain Co.: Blowing Springs Cave*, Flowstone Cave*, Lost Nantahala Cave*, Sand Cave*.

Comments: Litocampa diplurans are common in caves in Interior Low Plateau and Appalachians karst regions. Cave records from North Carolina may include multiple undescribed species.

RefERENCES: ${ }^{1}$ Christ (2003); * this study. 
Family Japygidae

Undetermined Genus

Undetermined species (TP/TX) A Two-pronged Bristletail

LoCALITIES: Polk Co.: Zig-Zag Fissure'.

COMmENTS: This family is reported rarely from caves in the eastern United States.

References: ${ }^{1}$ Christ (2003).

\section{Class Insecta \\ Order Coleoptera \\ Family Carabidae \\ Genus Scaphinotus}

\section{Scaphinotus andrewsi montanus (Valentine, 1935) (TX/AC) A Ground Beetle}

LOCALITIES: Avery Co.: Davis Moonshine Cave*.

Conservation Status: IUCN: Not assessed; NatureServe: Not assessed.

COMMENTS: This large beetle was observed dragging its preferred food (a snail) across the cave floor.

REFERENCES: * this study.

Family Coccinellidae

Undetermined Genus

Undetermined species (AC) A Ladybug

LOCALITIES: Rutherford Co.: Rumbling Bald Cave*.

REFERENCES: * this study.

\section{Family Staphylinidae \\ Genus Lathrobium}

\section{Lathrobium sp. (TX) A Rove Beetle}

LocAlities: Jackson Co.: Balsam Cave*.

REFERENCES: * this study.

\section{Genus Leptusa}

\section{Leptusa cribratula (Casey, 1906) (TX) A Rove Beetle}

LocAlities: Henderson Co.: Stillers Cave*.

Conservation Status: IUCN: Not assessed; NatureServe: GNR (SNR in North Carolina).

RefERENCES: * this study.

\section{Undetermined Genus}

Undetermined species (TX/AC) A Rove Beetle

LocAlITIES: McDowell Co.: Linville Caverns*.

REFERENCES: * this study.

\section{Order Diptera \\ Family Culicidae \\ Genux Culex}

\section{Culex sp. (TX) A Mosquito}

LocAlitIES: McDowell Co.: Linville Caverns*.

COMmENTS: Mosquitoes often overwinter in caves.

REFERENCES: * this study.

\section{Family Heleomyzidae \\ Undetermined Genus}

\section{Undetermined species (TX) A Sunfly}

LOCALITIES: Swain Co.: Blowing Springs Cave*.

COMmENTS: Heleomyzid flies are common in caves, particularly in winter. The two most common species in caves in the eastern United States are Amoebaleria defessa and Oecothea specus.

REFERENCES: * this study. 


\section{Family Limoniidae \\ Genus Chionea}

Chionea scita Walker, 1848 (TX) A Crane Fly

LOCALITIES: Rutherford Co.: Amazing Bat Cave1.

Conservation Status: IUCN: Not assessed; NatureServe: Not assessed.

COMmENTS: Crane flies are observed on occasion in the twilight zones of caves.

REFERENCES: ${ }^{1}$ Gaddy (1986a).

\section{Chionea sp. (TX) A Crane Fly}

LOCALITIES: Rutherford Co.: Bat Cave complex ${ }^{1}$.

COMmENTS: Crane flies are observed on occasion in the twilight zones of caves.

RefERENCEs: ${ }^{1}$ Hertl (1981).

\section{Family Muscidae \\ Undetermined Genus}

Undetermined species (TX/AC) A House Fly

LOCALITIES: Rutherford Co.: Bat Cave complex'.

RefERENCES: ${ }^{1}$ Hertl (1981).

\section{Family Mycetophilidae Genus Orfelia}

\section{Orfelia fultoni (Fisher, 1940) (TX) Foxfire Fly}

Localities: Burke Co.: Conley Cove Rockhouse*, Glow Worm Grotto*; McDowell Co.: Lake Tahoma Cave*. Conservation Status: IUCN: Not assessed; NatureServe: Not assessed.

COMmENTS: This bioluminescent fly was found near the entrances and twilight zones of these three caves.

REFERENCES: * this study.

\section{Undetermined Genus}

\section{Undetermined species (TX) A Hump-backed Fly}

LoCALITIES: Swain Co.: Blowing Springs Cave*.

COMMENTS: Mycetophilid flies are reported frequently from caves.

REFERENCES: * this study.

\section{Family Psychodidae \\ Undetermined Genus}

Undetermined species (TX/AC) A Moth Fly

LOCALITIES: Rutherford Co.: Bat Cave complex ${ }^{1}$.

REFERENCES: ${ }^{1}$ Hertl (1981).

\section{Family Sciaridae \\ Undetermined Genus}

Undetermined species (TP/TX) A Dark-winged Fungus Gnat

LOCALITIES: Rutherford Co.: Bat Cave complex'1.

COMMENTS: Sciarid flies are reported frequently from caves.

RefERENCES: ${ }^{1}$ Hertl (1981).

\section{Order Ephemeroptera \\ Family Heptageniidae \\ Genus Stenonema}

Stenonema sp. (AC) A Mayfly

LocAlitIES: Onslow Co.: Brinson's Rockhouse Cave*.

REFERENCES: * this study. 


\section{Order Hemiptera \\ Family Cicadellidae \\ Genus Erythroneura}

Erythroneura sp. (AC) A Leafhopper

LoCALITIES: Rutherford Co.: Block Creep Cave'.

References: ${ }^{1}$ Christ (2003).

\section{Family Reduviidae \\ Genus Empicoris}

\section{Empicoris sp. (AC) A Thread-legged Bug}

LoCALITIES: Rutherford Co.: Block Creep Cave'1.

References: ${ }^{1}$ Christ (2003).

\section{Order Hymenoptera}

Family Braconidae

Undetermined Genus

Undetermined species (AC) A Parasitoid Wasp

LOCALITIES: Rutherford Co.: Bat Cave complex1.

REFERENCES: ${ }^{1}$ Hertl (1981).

\section{Family Formicidae \\ Undetermined Genus}

Undetermined species (AC) An Ant

LOCALITIES: Rutherford Co.: Dusty Bat Cave*.

REFERENCES: * this study.

\section{Order Lepidoptera \\ Family Erebidae \\ Genus Scoliopteryx}

Scoliopteryx libatrix (Linnaeus, 1758) (TX) Herald Moth

LocAlities: Ashe Co.: Three Top Mountain Wildcat Den*; Avery Co.: Cranberry Mines*; Burke Co.: Cardboard Cave*; Henderson Co.: Cloven Cliffs Cave*, Devils Kitchen Cave*; McDowell Co.: Bennett's Mill Cave*; Rutherford Co.: Breakdown Cave*; Stokes Co.: Scoliopteryx Column Cave*.

Conservation Status: IUCN: Not assessed; NatureServe: G5 (SNR in North Carolina).

COMMENTS: This species commonly overwinters in caves in eastern North America.

REFERENCES: * this study.

\section{Order Neuroptera \\ Family Myrmeleontidae \\ Undetermined Genus}

Undetermined species (AC) An Antlion

LocAlitIES: Henderson Co.: Indian Cave*.

REFERENCES: * this study.

\section{Order Orthoptera}

Family Rhaphidophoridae

Genus Ceuthophilus

\section{Ceuthophilus sp. (TX) A Camel Cricket}

LoCAlitIES: McDowell Co.: Staircase Cave*, Limekiln Cave*.

COMMENTS: These records are likely $C$. gracilipes.

REFERENCES: * this study. 


\section{Genus Euhadenoecus}

\section{Euhadenoecus adelphus Hubbell \& Norton, 1978 (TX) A Cave Cricket}

LocAltTIES: Macon Co.: Granite City Cave*; Rutherford Co.: Bat Cave complex ${ }^{1,2}$; Swain Co.: Blowing Springs Cave*.

Conservation Status: IUCN: Not assessed; NatureServe: GNR (SNR in North Carolina).

COMMENTS: Cave crickets were commonly observed in many caves in North Carolina. However, only confirmed records are presented.

References: ${ }^{1}$ Hubbell and Norton (1978), ${ }^{2}$ Hertl (1981), * this study.

\section{Euhadenoecus puteanus (Scudder, 1877) (TX) Puteanus Camel Cricket}

Localities: Madison Co.: Campbells Vegetable Cave'; McDowell Co.: Linville Caverns ${ }^{1,2}$; Swain Co.: cave in Indian Ridge near Judson².

Conservation Status: IUCN: Not assessed; NatureServe: Not assessed.

COMmENTS: Cave crickets were commonly observed in many caves in North Carolina. However, only confirmed records are presented.

References: ${ }^{1}$ Petrie (1942), ${ }^{2}$ Hubbell and Norton (1978).

Euhadenoecus sp. (TX) A Cave Cricket

LocAlities: Madison Co.: Anthodite Cave*; McDowell Co.: Staircase Cave*; Rutherford Co.: Breakdown Cave*, Moonshiners Cave*; Transylvania Co.: Snake Den no. 2 Cave*; Yancey Co.: Celo Cave*.

Comments: Cave crickets were commonly observed in many caves in North Carolina. However, only confirmed records are presented. These records are either E. adelphus or E. puteanus.

REFERENCES: * this study.

\section{Order Zygentoma \\ Family Lepismatidae \\ Undetermined Genus}

\section{Undetermined species (TX/AC) A Silverfish}

Localities: McDowell Co: Wind Cave*; Rutherford Co.: Breakdown Cave*, Rumbling Bald Cave*; Swain Co.: Blowing Springs Cave*.

REFERENCES: * this study.

\section{Subphylum Crustacea \\ Class Malacostraca \\ Order Amphipoda \\ Family Crangonyctidae \\ Genus Stygobromus}

\section{Stygobromus araeus (Holsinger, 1969) (SB) Tidewater Interstitial Amphipod}

LOCALITIES: Gates Co.: seepage spring near Merchants Mill Pond ${ }^{1,2}$.

Conservation StATUS: IUCN: Vulnerable D2 (Inland Water Crustacean Specialist Group 1996a); NatureServe: G3 (SU in North Carolina).

COMMENTS: This small groundwater amphipod also is known from several seepage springs and seep-fed streams in the Coastal Plain of Virginia (Culver et al., 2012).

RefERENCES: ${ }^{1}$ Terwilliger (1991); ${ }^{2}$ Culver et al. (2012).

\section{Stygobromus carolinensis Holsinger, 1978 (SB) Carolina Groundwater Amphipod}

LoCALITIES: Yancey Co.: seeps on W side of State Rd 128, $2.4 \mathrm{~km} \mathrm{~N}$ of Blue Ridge Parkway (type locality) 1.

Conservation Status: IUCN: Not assessed; NatureServe: G1 (S1 in North Carolina).

COMMENTS: This small groundwater amphipod is known only from the type locality.

REFERENCES: ${ }^{1}$ Holsinger (1978).

\section{Stygobromus cf. carolinensis (SB) A Cave Amphipod}

LOCALITIES: Wilkes Co.: Harrold Mountain Cave*.

COMmENTS: Specimens from Harrold Mountain Cave may be S. carolinensis or an undescribed species.

REFERENCES: * this study. 


\section{Stygobromus indentatus (Holsinger, 1967) (SB) Tidewater Amphipod}

LoCALITIES: Nash Co.: shallow well at Bailey ${ }^{1,2}$.

Conservation Status: IUCN: Vulnerable D2 (Inland Water Crustacean Specialist Group, 1996b); NatureServe: G3 (SU in North Carolina).

COMmENTS: This relatively large groundwater amphipod also is known from several seepage springs and wells in the Coastal Plain of Maryland and Virginia (Culver et al., 2012).

ReferenCES: ${ }^{1}$ Holsinger (1978); ${ }^{2}$ Culver et al. (2012).

\section{Stygobromus nov. sp. A (SB) A Cave Amphipod}

LoCALITIES: Avery Co.: Black Rocks Mystery Cave*, Charlie’s Ridge Bat Cave*, Frying Pan Cave*; Burke Co.: Odell Cave $^{*}$; Henderson Co.: Stillers Cave*; McDowell Co.: Lake Tahoma Cave*, Linville Caverns*, Roadside Surprise Cave*, Staircase Cave*, Wind Cave*; Mitchell Co.: Buckshot Cave*.

COMMENTS: This undescribed species is the most wide-ranging groundwater amphipod in North Carolina.

REFERENCES: * this study.

\section{Stygobromus nov. sp. B (SB) A Cave Amphipod}

LOCALITIES: Jackson Co.: seep below Jones Knob1.

Conservation Status: IUCN: not assessed; NatureServe: GNR (S1? in North Carolina).

COMMENTS: This undescribed groundwater amphipod is known only from a seep in Jackson County.

REFERENCES: ${ }^{1}$ NCNHP.

\section{Stygobromus sp. (SB) A Cave Amphipod}

LoCALITIES: Buncombe Co.: Cedar Cliff Cave*; McDowell Co.: Roadside Surprise Cave*, Staircase Cave*; Polk Co.: Little Warrior Mountain Cave*.

COMMENTS: These records represent collections of immatures or females that could not be identified to species.

REFERENCES: * this study.

\section{Order Decapoda \\ Family Cambaridae \\ Genus Cambarus}

\section{Cambarus asperimanus Faxon, 1914 (SX) Mitten Crayfish}

LocAlITIES: McDowell Co.: Staircase Cave1.

Conservation Status: IUCN: Least concern; NatureServe: G4 (S4 in North Carolina).

REFERENCES: ${ }^{1}$ NCSM.

\section{Cambarus bartonii (Fabricius, 1798) (SP) Common Crayfish}

LoCALITIES: McDowell Co.: Linville Caverns ${ }^{1,2}$, Staircase Cave'.

Conservation Status: IUCN: Least concern; NatureServe: G5 (S5 in North Carolina).

Comments: This species is common in caves in the Appalachian Valley and Ridge (Fong et al., 2012).

REFERENCES: ${ }^{1}$ NCSM; ${ }^{2}$ SCAN: USNM.

\section{Cambarus latimanus (LeConte, 1856) (SX) Variable Crayfish}

LoCALITIES: Jones Co.: small limestone cave along NC 410.9 miles west of Comfort; Onslow Co.: small limestone cave off SR 12222 miles NNE of Catherine Lake'.

Conservation Status: IUCN: Least concern; NatureServe: G5 (S5 in North Carolina).

REFERENCES: ${ }^{1}$ NCSM.

\section{Genus Procambarus}

\section{Procambarus acutus (Girard, 1852) (SX) White River Crayfish}

LocALITIES: Onslow Co.: small limestone cave off SR 12222 miles NNE of Catherine Lake'.

Conservation Status: IUCN: Least concern; NatureServe: G5 (S5 in North Carolina).

RefERENCES: ${ }^{1}$ NCSM.

\section{Order Isopoda \\ Family Armadillidiidae \\ Genus Armadillidium}

\section{Armadillidium vulgare Latreille, 1804 (TP) Common Woodlouse}

LoCALITIES: Henderson Co.: Hog Rock Cave'; Rutherford Co.: Tank Bug Cave*. 
Conservation Status: IUCN: Not assessed; NatureServe: GNR (SNR in North Carolina).

Comments: This species is native to Europe but has been introduced throughout North America and is often observed in caves.

References: ${ }^{1}$ Christ (2003), * this study.

\section{Family Asellidae \\ Genus Caecidotea}

\section{Caecidotea carolinensis Lewis \& Bowman, 1977 (SB) Bennett's Mill Cave Isopod}

LocAlITIES: McDowell Co.: Bennetts Mill Cave (type locality) ${ }^{1-3}$.

Conservation Status: IUCN: Not assessed; NatureServe: G2G3 (SH in North Carolina; Listed as Endangered in North Carolina (NCWRC 2017).

Comments: This stygobite also has been collected from Parlar Cave in Orangeburg Co., South Carolina (Reeves, 2000a) suggesting that this species may be more widespread than currently known.

REFERENCES: ${ }^{1}$ NCNHP; ${ }^{2}$ SCAN: USNM; ${ }^{3}$ Lewis and Bowman (1977).

\section{Family Trichoniscidae Genus Miktoniscus}

\section{Miktoniscus linearis (Patience, 1908) (TP) A Terrestrial Isopod}

LOCALITIES: McDowell Co.: Linville Caverns ${ }^{1,2}$.

Conservation Status: IUCN: Not assessed; NatureServe: Not assessed.

Comments: This species was described in England; however, Schultz (1964) compared three specimens collected from Linville Caverns with specimens redescribed by Vandel (1950) and noted that they were identical.

REFERENCES: ${ }^{1}$ SCAN: USNM; ${ }^{2}$ Schultz (1964).

\section{Class Maxillopoda \\ Order Cyclopoida \\ Family Cyclopidae \\ Genus Cyclops}

\section{Cyclops sp. (SP/SX) A Copepod}

LOCALITIES: McDowell Co.: Staircase Cave*.

REFERENCES: * this study.

\section{Genus Diacyclops}

Diacyclops crassicaudis brachycercus (Kiefer, 1929) (SP) A Copepod

LOCALITIES: Chatham Co.: well 200 yards south of the Orange-Chatham county line'; well 12 miles east of Pittsboro'.

Conservation Status: IUCN: Not assessed; NatureServe: Not assessed.

Comments: This wide-ranging species in North America is occasional sampled from groundwater habitats (Reid, 2004; Lewis and Reid, 2007).

REFERENCES: ${ }^{1}$ Yeatman (1943).

\section{Diacyclops jeanneli putei (Yeatman, 1943) (SB) Carolina Well Copepod}

LoCALITIES: Orange Co.: well 2.7 miles from the Wilson Zoological Laboratory on US 501 (type locality) 1.

Conservation Status: IUCN: Not assessed; NatureServe: G3G4 (SH in North Carolina); Listed as Special Concern in North Carolina (NCWRC, 2017).

COMMENTS: This subspecies is only known from North Carolina.

REFERENCES: ${ }^{1}$ Yeatman (1943).

\section{Diacyclops navus (Herrick, 1882 (SP) A Copepod}

LocalitIES: Chatham Co.: well 12 miles east of Pittsboro'; well 2 mi from US 501 between Pittsboro and Moncure'; well 2.5 north of US 1 near New Elam Christian Church1.

Conservation Status: IUCN: Not assessed; NatureServe: Not assessed.

COMmENTS: This species also has been collected from groundwater habitats in Indiana (Lewis and Reid, 2007).

RefERENCES: ${ }^{1}$ Yeatman (1943). 


\section{Diacyclops nearcticus (Kiefer, 1934) (SP) A Copepod}

LOCALITIES: Chatham Co.: well 1.5 miles north of Fearrington'.

Conservation Status: IUCN: Not assessed; NatureServe: G3G4 (SNR in North Carolina).

COMmENTS: This species ranges throughout the eastern and central United States. It also has been collected from groundwater habitats in Florida, Indiana, Kentucky, and Tennessee (Strayer and Reid, 1999; Bruno et al., 2000; Reid, 2004; Lewis and Reid, 2007).

ReferenCES: ${ }^{1}$ Yeatman (1943).

\section{Genus Eucyclops}

\section{Eucyclops serrulatus (Fischer, 1851) (SX) A Copepod}

LOCALITIES: Orange Co.: well 2.7 miles from the Wilson Zoological Laboratory on US $501^{1}$.

Conservation Status: IUCN: Not assessed; NatureServe: Not assessed.

Comments: This species also is known from Mammoth Cave in Kentucky (Kofoid, 1900).

REFERENCES: ${ }^{1}$ Yeatman (1943).

\section{Genus Microcyclops}

\section{Microcyclops rubellus (Lilljeborg, 1901) (SP) A Copepod}

LOCALITIES: Chatham Co.: well 12 miles east of Pittsboro1.

Conservation Status: IUCN: Not assessed; NatureServe: Not assessed.

COMmENTS: This species also is known from groundwater habitats in Illinois, Indiana, Kentucky, and Tennessee (Strayer and Reid, 1999; Lewis and Reid, 2007).

REFERENCES: ${ }^{1}$ Yeatman (1943).

\section{Subphylum Myriapoda \\ Class Chilopoda \\ Order Lithobiomorpha \\ Family Lithobiidae \\ Genus Watobius}

\section{Watobius anderisus Chamberlin, 1911 (AC) A Centipede}

Localities: Henderson Co.: Hog Rock Cave ${ }^{1}$; Rutherford Co.: McGrath Fissure ${ }^{1}$, Western Talus Tunnel'

Conservation Status: IUCN: Not assessed; NatureServe: Not assessed.

RefERENCES: ${ }^{1}$ Christ (2003).

\section{Order Scolopendromorpha \\ Family Scolopocryptopidae \\ Genus Scolopocryptops}

Scolopocryptops sp. (AC) A Centipede

LoCALITIES: Rutherford Co.: Western Talus Tunnel'1.

Conservation Status: na.

References: ${ }^{1}$ Christ (2003).

\section{Class Diplopoda \\ Order Chordeumatida \\ Family Cleidogonidae \\ Genus Pseudotremia}

\section{Pseudotremia fracta nantahala Hoffman, 1981 (TX) A Millipede}

LocAlITIES: Swain Co.: Blowing Springs Cave (type locality) 1 .

Conservation Status: IUCN: Not assessed; NatureServe: Not assessed.

COMmENTS: This pigmented species is known only from the type locality and from Cliff Ridge near Blowing Springs.

References: ${ }^{1}$ Hoffman (1981).

\section{Pseudotremia shelleyi Lewis, 2009 (TB) Shelley's Cave Millipede}

LocAlities: McDowell Co.: Lime Kiln Cave ${ }^{3}$; Rutherford Co.: Bat Cave complex ${ }^{1,3}$; Rumbling Bald Cave (type locality) ${ }^{3,4}$;

Rumbling Bald Spring Cave ${ }^{3,4}$.

Conservation Status: IUCN: Not assessed; NatureServe: Not assessed. 
Comments: This is the only troglobitic Pseudotremia in North Carolina. Several troglobitic Pseudotremia are known from the Appalachians and Interior Low Plateau karst regions (Lewis, 2000, 2005b, 2009; Shear, 2008, 2011).

RefERENCES: ${ }^{1}$ Hertl (1981); ${ }^{2}$ Shelley (2000); ${ }^{3}$ Lewis (2009), ${ }^{4}$ NCSM.

\section{Pseudotremia sp. (TP/TX) A Millipede}

LocAlitIES: Avery Co.: Cranberry Mines²; Rutherford Co.: A-Frame Fissure ${ }^{3}$, Bat Cave complex ${ }^{1,2}$; Sliding Rock Cave ${ }^{3}$; Madison Co.: Anthodite Cave'2.

COMMENTS: Specimens from these caves could not be identified to species.

References: ${ }^{1}$ Hertl (1981); ${ }^{2}$ Shelley (2000); ${ }^{3}$ Christ (2003).

\section{Family Trichopetalidae \\ Genus Nannopetalum}

\section{Nannopetalum vespertilio Shear, 2003 (TB) Bat Cave Millipede}

LOCALITIES: Rutherford Co.: Bat Cave (type locality)1.

Conservation Status: IUCN: Not assessed; NatureServe: GNR (SNR in North Carolina).

COMMENTS: This troglobite is known only from the type locality and is the smallest known trichopetalid millipede measuring under $4 \mathrm{~mm}$ in total length.

REFERENCES: ${ }^{1}$ Shear (2003).

\section{Order Julida \\ Family Parajulidae \\ Genus Ptyoiulus}

Ptyoiulus sp. (TX/AC) A Millipede

LoCALITIES: Rutherford Co.: Western Talus Tunnel*.

COMmENTS: This genus is known from several surface records in North Carolina (Shelley, 2000).

REFERENCES: * this study.

\section{Order Polydesmida \\ Family Macrosternodesmidae \\ Genus Chaetaspis}

Chaetaspis sp. (TX/AC) A Millipede

LocAlities: Swain Co.: Lost Nantahala Cave'.

COMMENTS: This genus is known primarily from surface records in North Carolina (Shelley, 2000).

REFERENCES: ${ }^{1}$ Shelley (2000).

\section{Family Polydesmidae \\ Genus Pseudopolydesmus}

Pseudopolydesmus sp. (TX/AC) A Millipede

LOCALITIES: Rutherford Co.: Western Talus Tunnel'1.

COMmENTS: This may be one of four species Pseudopolydesmus known primarily from surface records in North Carolina (Shelley, 2000).

REFERENCES: ${ }^{1}$ Christ (2003).

\section{Order Spirostreptida \\ Family Cambalidae \\ Genus Cambala}

\section{Cambala annulata (Say, 1821) (TX) A Millipede}

LocAliTIES: Henderson Co.: Hog Rock Cave'.

Conservation Status: IUCN: Not assessed; NatureServe: G5 (SNR in North Carolina).

COMmENTS: This species is known primarily from surface records in several counties in North Carolina (Shelley, 2000).

REFERENCES: ${ }^{1}$ Christ (2003).

Cambala hubrichti Hoffman, 1958 (TX) A Millipede

LocAliTIES: Rutherford Co.: Bat Cave complex'1. 
Conservation Status: IUCN: Not assessed; NatureServe: G5 (SNR in North Carolina).

COMMENTS: This species is known primarily from surface records in several counties in North Carolina (Shelley, 2000).

RefERENCES: ${ }^{1}$ Hertl (1981).

\section{Cambala sp. (TX) A Millipede}

LocAlitIES: McDowell Co.: Linville Caverns*; Rutherford Co.: McGrath Fissure'; Swain Co.: Blowing Springs Cave*.

COMMENTS: These records may be either $C$. annulata or $C$. hubrichti.

RefERENCES: ${ }^{1}$ Christ (2003); ${ }^{*}$ this study.

\section{Class Symphyla \\ Order Cephalostigmata \\ Family Scutigerellidae \\ Genus Scutigerella}

\section{Scutigerella sp. (TP/TX) A Symphylan}

Localities: Henderson Co.: Devils Kitchen Cave1; Rutherford Co.: Western Talus Tunnel'1.

COMMENTS: These soil-dwelling arthropods are not well represented in cave collections.

References: ${ }^{1}$ Christ (2003).

\section{Phylum Chordata \\ Subphylum Vertebrata \\ Class Actinopterygii \\ Order Cypriniformes \\ Family Cyprinidae \\ Genus Rhinichthys}

\section{Rhinichthys cataractae (Valenciennes, 1842) (AC) Longnose Dace}

LOCALITIES: McDowell Co.: Linville Caverns*.

Conservation Status: IUCN: Least Concern; NatureServe: G5 (S5 in North Carolina).

Comments: This species also has been reported from caves in Minnesota and West Virginia (Dearolf, 1956; Schmidt, 1994; Poly, 2001).

REFERENCES: * this study.

\section{Order Salmoniformes \\ Family Salmonidae \\ Genus Oncorhynchus}

Oncorhynchus mykiss (Walbaum, 1792) (AC) Rainbow Trout

LoCALITIES: McDowell Co.: Linville Caverns*.

Conservation Status: IUCN: Not assessed; NatureServe: G5 (SNA in North Carolina).

COMmENTS: Trout were purposefully stocked at Linville Caverns by the owners.

REFERENCES: * this study.

\section{Genus Salmo}

Salmo trutta Linnaeus, 1758 (AC) Brown Trout

LocAlitIES: McDowell Co.: Linville Caverns*.

Conservation Status: IUCN: Least Concern; NatureServe: G5 (SNA in North Carolina).

COMMENTS: Trout were purposefully stocked at Linville Caverns by the owners.

REFERENCES: * this study.

\section{Genus Salvelinus}

\section{Salvelinus fontinalis (Mitchill, 1814) (AC) Brook Trout}

Localities: McDowell Co.: Linville Caverns*.

Conservation Status: IUCN: Not assessed; NatureServe: G5 (S5 in North Carolina); Listed as a Species of Greatest Conservation Need in North Carolina (North Carolina Wildlife Resources Commission, 2015).

COMMENTS: Trout were purposefully stocked at Linville Caverns by the owners.

REFERENCES: * this study. 


\section{Class Amphibia \\ Order Caudata \\ Family Plethodontidae \\ Genus Aneides}

\section{Aneides caryaensis Patton et al., 2019 (TX) Green Salamander}

LOCALITIES: Henderson Co.: Green Salamander Shelter*.

Conservation Status: IUCN: Near Threatened (Hammerson 2004); NatureServe: G3G4 (S2S3 in North Carolina); Listed as Threatened in North Carolina (North Carolina Wildlife Resources Commission, 2017a); Listed as a Species of Greatest Conservation Need in North Carolina (North Carolina Wildlife Resources Commission, 2015). Conservation statuses are for $A$. aeneus, as this species was described last year and has not yet been assessed officially.

COMmENTS: This species is a member of the Green Salamander ( $A$. aeneus) species complex and was described recently (Patton et al. 2019). Aneides aeneus has been observed infrequently in the twilight zone and entrance area of caves throughout its range (Dearolf, 1956; Osbourn, 2005; M.L. Niemiller, unpublished data). We believe this occurrence to represent a new site for this newly described salamander endemic to Hickory Nut Gorge.

ReFERENCES: * this study.

\section{Genus Desmognathus}

\section{Desmognathus monticola Dunn, 1916 (TX/AC) Seal Salamander}

Localities: McDowell Co.: Linville Caverns*.

Conservation Status: IUCN: Least Concern; NatureServe: G5 (S5 in North Carolina).

Comments: Like other Desmognathus, D. monticola has been infrequently reported from caves throughout its range (Garton et al., 1993; Pauley, 1993; Osbourn, 2005).

REFERENCES: * this study.

\section{Desmognathus sp. (TX/AC) A Dusky Salamander}

LoCALITIES: Rutherford Co.: Rumbling Bald Spring Cave*, White Root Shelter*.

COMmENTS: Desmognathus salamanders are infrequently reported from caves in the Blue Ridge Mountains and adjacent Appalachian Valley and Ridge, with most records from the entrance or twilight zones (Wallace, 1984, 2003; Garton et al., 1993; Osbourn, 2005; Niemiller et al., 2016).

REFERENCES: * this study.

\section{Genus Eurycea}

\section{Eurycea wilderae Dunn, 1920 (TX/AC) Blue Ridge Two-lined Salamander}

LocAlities: McDowell Co.: Linville Caverns*.

Conservation Status: IUCN: Least Concern; NatureServe: G5 (S5 in North Carolina).

COMmENTS: Eurycea wilderae has been reported from a few caves in eastern Tennessee, including Great Smoky Mountains National Park (Wallace, 1984, 2003; Dodd et al., 2001; Niemiller et al., 2016).

REFERENCES: * this study.

\section{Genus Gyrinophilus}

\section{Gyrinophilus porphyriticus (Green, 1827) (TP) Spring Salamander}

LOCALITIES: Rutherford Co.: White Root Shelter*.

Conservation Status: IUCN: Least Concern; NatureServe: G5 (S5 in North Carolina).

COMmEnTS: This species is common in caves in the Appalachian Valley and Ridge and Blue Ridge Mountains (Dearolf, 1956; Brandon, 1966; Cooper and Cooper, 1968; Wallace, 1984, 2003; Garton et al., 1993; Dodd et al., 2001; Osbourn, 2005; Miller and Niemiller, 2008; Miller et al., 2008; Niemiller and Miller, 2009; Niemiller et al., 2010).

RefERENCES: * this study.

\section{Genus Plethodon}

\section{Plethodon cinereus (Green, 1818) (TX/AC) Eastern Red-backed Salamander}

LOCALITIES: McDowell Co.: Linville Caverns*.

Conservation Status: IUCN: Least Concern; NatureServe: G5 (S5 in North Carolina).

Comments: This species also has been reported from caves in Indiana, Maryland, Virginia, and West Virginia (Dearolf, 1956; Garton et al., 1993; Osbourn, 2005).

REFERENCES: * this study. 
Plethodon cylindraceus (Harlan, 1825) (TX) White-spotted Slimy Salamander

Localities: McDowell Co.: Linville Caverns*; Rutherford Co.: Breakdown Cave*, Rimstone Skull Cave*.

Conservation Status: IUCN: Least Concern; NatureServe: G5 (S5 in North Carolina).

Comments: Plethodon cylindraceus is a member of the Slimy Salamander ( $P$. glutinosus) species complex. A few members of this complex, such as. $P$. glutinosus and $P$. albagula, are common in caves throughout their respective ranges (Garton et al., 1993; Buhlmann, 2001; Trauth et al., 2004; Taylor and Mays, 2006; Taylor et al., 2015; Niemiller et al., 2016).

REFERENCES: * this study.

Plethodon metcalfi Brimley, 1912 (TX) Southern Gray-cheeked Salamander

LOCALITIES: Polk Co.: Little Warrior Mountain Cave1.

Conservation Status: IUCN: Least Concern; NatureServe: G4 (S5 in North Carolina).

Comments: Plethodon metcalfi is a member of the $P$. jordani species complex. Gaddy (1986a) observed this species (reported as $P$. jordani) at the entrance to Little Warrior Mountain Cave. Highton and Peabody (2000) removed $P$. metcafi from synonymy with $P$. jordani.

ReferenCES: ${ }^{1}$ Gaddy (1986a).

\title{
Plethodon yonahlossee Dunn, 1917 (TX) Yonahlossee Salamander
}

Localities: McDowell Co.: Limekiln Cave*, Linville Caverns*, Wind Cave*; Rutherford Co.: Bat Cave complex ${ }^{2,3}$, Breakdown Cave*, Rumbling Bald Cave*; Watauga Co.: cave along Howard's Creek'.

Conservation Status: IUCN: Least Concern; NatureServe: G4 (S4 in North Carolina); Listed as a Species of Greatest Conservation Need in North Carolina (North Carolina Wildlife Resources Commission, 2015).

Comments: Plethodon yonahlossee from the Bat Cave area in Rutherford County are a unique variant, commonly referred to as the Crevice Salamander. This species is often found in rock crevices (Beane et al., 2010; Niemiller and Reynolds, 2011).

RefERENCES: ${ }^{1}$ VertNet: UF; ${ }^{2}$ NCSM; ${ }^{3}$ Holland (1981); ${ }^{*}$ this study.

\section{Genus Pseudotriton}

\section{Pseudotriton ruber (Latreille, 1801) (TP) Red Salamander}

Localities: McDowell Co.: Linville Caverns*.

Conservation Status: IUCN: Least Concern; NatureServe: G5 (S5 in North Carolina).

COMmENTS: This species has been reported from several caves in the Interior Low Plateau and Appalachian Valley and Ridge to the west of the study area where it is frequently found in the twilight zone (Buhlmann, 2001; Osbourn, 2005; Camp and Jensen, 2007; Godwin, 2008; Miller et al., 2008; Niemiller and Miller, 2009; Niemiller and Reeves, 2014; Niemiller et al., 2016). Pseudotriton ruber has been documented to breed in caves (Miller and Niemiller, 2005; Niemiller et al., 2006; Miller et al., 2008).

REFERENCES: * this study.

\author{
Class Reptilia \\ Order Squamata \\ Suborder Serpentes \\ Family Colubridae \\ Genus Thamnophis
}

Thamnophis sirtalis (Linnaeus, 1766) (AC) Common Gartersnake

LoCALITIES: Wake Co.: Raleigh Mine'.

Conservation Status: IUCN: Least Concern; NatureServe: G5 (S5 in North Carolina).

Comments: This species also has been reported from caves in Pennsylvania and Tennessee (Dearolf, 1956; Niemiller et al., 2016).

REFERENCES: ${ }^{1}$ VertNet: USNM.

\section{Family Dipsadidae \\ Genus Diadophis}

\section{Diadophis punctatus (Linnaeus, 1766) (AC) Ring-necked Snake}

LoCAlities: Burke Co.: Holy Moley Cave*.

Conservation Status: IUCN: Least Concern; NatureServe: G5 (S5 in North Carolina). 
Comments: This species has been reported on occasion from caves (Brode, 1958; Cliburn and Middleton, 1983; Pauley, 1993; Niemiller et al., 2016).

REFERENCES: * this study.

\section{Family Viperidae \\ Genus Agkistrodon}

Agkistrodon contortrix (Linnaeus, 1766) (AC) Copperhead

LocAlities: Burke Co.: Copperhead Cave*; Davidson Co.: New Boone's Cave*.

Conservation Status: IUCN: Least Concern; NatureServe: G5 (S5 in North Carolina).

COMmENTS: This species is observed on occasion around cave entrances (Dearolf, 1956; Pauley, 1993; Dodd et al., 2001; Niemiller et al., 2016).

REFERENCES: * this study.

\section{Genus Crotalus}

\section{Crotalus horridus Linnaeus, 1758 (AC) Timber Rattlesnake}

LoCALITIES: McDowell Co.: Crotalus Shelter*; Polk Co.: Rattlesnake Fissure*.

Conservation Status: IUCN: Least Concern; NatureServe: G4 (S3 in North Carolina); Listed as Special Concern in North Carolina (North Carolina Wildlife Resources Commission, 2017a); Listed as a Species of Greatest Conservation Need in North Carolina (North Carolina Wildlife Resources Commission, 2015).

COMMENTS: This species is observed on occasion around cave entrances (Dearolf, 1956; Garton et al., 1993; Osbourn, 2005).

REFERENCES: * this study.

\section{Suborder Lacertilia \\ Family Dactyloidae \\ Genus Anolis}

\section{Anolis carolinensis (Voigt, 1832) (AC) Green Anole}

LoCALITIES: Rutherford Co.: Rumbling Bald Cave*.

Conservation Status: IUCN: Least Concern; NatureServe: G5 (S5 in North Carolina).

COMMENTS: Lizards, including anoles, are not regularly encountered in caves, and all records are suspected to be accidental occurrences.

REFERENCES: * this study.

\section{Class Aves \\ Order Passeriformes \\ Family Corvidae \\ Genus Corvus}

Corvus corax Linnaeus, 1758 (TX/AC) Common Raven

LocAlities: Yancey Co.: Cooper's Cave*.

Conservation Status: IUCN: Least Concern; NatureServe: G5 (S3 in North Carolina); Listed as a Species of Greatest Conservation Need in North Carolina (North Carolina Wildlife Resources Commission, 2015).

COMmENTS: We observed a pair of Common Ravens nesting at a skylight entrance to Cooper's Cave in March 2006.

REFERENCES: * this study.

\section{Family Tyrannidae \\ Genus Sayornis}

\section{Sayornis phoebe (Latham, 1790) (TX) Eastern Phoebe}

Localities: Avery Co.: Shelter of the Hands*; McDowell Co.: Staircase Cave*; Yancey Co.: Cooper's Cave*; Watauga Co.: Boone Rockhouse*.

Conservation Status: IUCN: Least Concern; NatureServe: G5 (S5 in North Carolina).

COMmEnTS: Sayornis phoebe is common throughout the eastern United States and is frequently encountered nesting on ledges in the entrances and twilight zone of caves (Dearolf, 1956; Garton et al., 1993; Fong et al., 2012; Niemiller et al., 2013, 2016; Slay et al., 2016).

REFERENCES: * this study. 


\section{Class Mammalia \\ Order Carnivora \\ Family Procyonidae \\ Genus Procyon}

Procyon lotor (Linnaeus, 1758) (TX) Raccoon

Localities: McDowell Co.: Wind Cave*.

Conservation Status: IUCN: Least Concern; NatureServe: G5 (S5 in North Carolina).

COMmENTS: Raccoons are known to enter caves throughout the range of the species (Dearolf, 1956; Garton et al., 1993; Niemiller et al. 2016). The authors recall observing raccoon scat in several North Carolina caves.

REFERENCES: * this study.

\section{Order Chiroptera \\ Family Vespertilionidae \\ Genus Corynorhinus}

\section{Corynorhinus rafinesquii Handley, 1955 (TX) Rafinesque's Big-eared Bat}

LoCALITIES: Swain Co.: Eagle Creek Mine', Sugar Fork Mine'; Yancey Co.: Cooper's Cave*.

Conservation Status: IUCN: Least Concern; NatureServe: G3G4 (S3 in North Carolina); Listed as Threatened in North Carolina (North Carolina Wildlife Resources Commission, 2017a); Listed as a Species of Greatest Conservation Need in North Carolina (North Carolina Wildlife Resources Commission, 2015).

COMmENTS: This species frequents caves throughout its range, primarily during winter. Populations in the western part of the state associated with mines and occasionally caves are considered C. r. rafinesquii.

References: ${ }^{1}$ Bat Population Database; * this study.

\section{Corynorhinus townsendii virginianus Handley, 1955 (TX) Virginia Big-eared Bat}

LocAlities: Avery Co.: Black Coral Cave ${ }^{1}$, Black Rock Cliffs Cave ${ }^{1-3}$, Black Rock Mystery Cave ${ }^{1}$, Pilot Knob Cave no. $1^{1}$, Thunder Hole ${ }^{1}$, Tom Terrific Cave'1.

Conservation Status: IUCN: Least Concern; NatureServe: G3G4T2 (S1 in North Carolina); Listed as Endangered in North Carolina (North Carolina Wildlife Resources Commission, 2017a); Listed as Endangered under the U.S. Endangered Species Act; Listed as a Species of Greatest Conservation Need in North Carolina (North Carolina Wildlife Resources Commission, 2015).

Comments: This federally endangered species is rare in North Carolina where it has been reported from caves and mines at high elevations in the western part of the state. The IUCN Red List status rank reflects the conservation status of the species and not this subspecies.

References: ${ }^{1}$ Boynton et al. (1992); ${ }^{2}$ VertNet: MVZ; ${ }^{3}$ Bat Population Database.

\section{Genus Eptesicus}

\section{Eptesicus fuscus (Beauvois, 1796) (TX) Big Brown Bat}

LocAlities: Avery Co.: Cranberry Mines*; Jackson Co.: Kitchen Cave ${ }^{1}$; Madison Co.: French Broad Cave*; McDowell Co.: Linville Caverns*; Rutherford Co.: Rumbling Bald Cave*, Sliding Rock Cave*; Transylvania Co.: Snake Den no. 2 Cave*; Yancey Co.: Cooper's Cave*, Isom Mica Mine*.

Conservation Status: IUCN: Least Concern; NatureServe: G5 (S5 in North Carolina).

Comments: Eptesicus fuscus is encountered frequently in caves throughout its range, particularly in winter, where it often roosts alone or in small clusters on ledges or in crevices.

REFERENCES: ${ }^{1}$ VertNet: MVZ; ${ }^{*}$ this study.

\section{Genus Myotis}

\section{Myotis leibii (Audubon \& Bachman, 1842) (TX) Eastern Small-footed Bat}

LocAlities: Avery Co.: Black Rock Cliffs Cave ${ }^{1,3}$; Jackson Co.: Kitchen Cave ${ }^{2}$; McDowell Co.: Wind Cave'; Rutherford Co.: Bat Cave ${ }^{1,3}$, Breakdown Cave1, Rumbling Bald Cave'; Swain Co.: Blowing Springs Cave1, Flowstone Cave*, Lost Nantahala Cave'; Yancey Co.: Cooper's Cave1; Isom Mica Mine*.

Conservation Status: IUCN: Endangered A4bce (Solari, 2018a); NatureServe: G4 (S2 in North Carolina); Listed as Special Concern in North Carolina (North Carolina Wildlife Resources Commission, 2017a); Listed as a Species of Greatest Conservation Need in North Carolina (North Carolina Wildlife Resources Commission, 2015). 
COMMENTS: This species is known primarily from mountainous areas in the western part of the state. The conservation status of M. leibii was elevated recently to Endangered due to impacts from White-nose Syndrome among other factors (Solari, 2018a).

References: ${ }^{1}$ Boynton et al. (1992); ${ }^{2}$ VertNet: MVZ; ${ }^{3}$ Bat Population Database, * this study.

\section{Myotis lucifugus (LeConte, 1831) (TX) Little Brown Bat}

Localities: Avery Co.: Black Rock Cliffs Cave², Cranberry Mines*; Jackson Co.: Kitchen Cave'; McDowell Co.: Linville Caverns*, Wind Cave*; Rutherford Co.: Bat Cave ${ }^{1}$, Moonshiners Cave*, Sliding Rock Cave*; Swain Co.: Blowing Springs Cave*; Yancey Co.: Celo Cave*, Isom Mica Mine*.

Conservation Status: IUCN: Endangered A3be (Solari, 2018b); NatureServe: G3 (S2 in North Carolina); Listed as a Species of Greatest Conservation Need in North Carolina (North Carolina Wildlife Resources Commission, 2015).

COMments: This species is known from all physiographic regions in North Carolina but is known primarily from mountainous areas in the western part of the state. Due to population declines associated with White-nose Syndrome, $M$. lucifugus is now considered Endangered by IUCN. Population declines have been particularly severe in North Carolina (North Carolina Wildlife Resources Commission, 2017b; O'Keefe et al., 2019). Declines also have been noted for some eastern Tennessee populations (Flock, 2013, 2014). This species is under review by USFWS for potential listing.

REFERENCES: ${ }^{1}$ VertNet: MVZ; ${ }^{2}$ VertNet: NCSM; ${ }^{*}$ this study.

\section{Myotis septentrionalis (Trovessart, 1897) (TX) Northern Long-eared Bat}

LoCAlities: Avery Co.: Black Rock Cliffs Cave ${ }^{1,3}$, Cranberry Mines ${ }^{*}$; Rutherford Co.: Bat Cave ${ }^{1}$ Rumbling Bald Cave ${ }^{3}$; Swain Co.: Blowing Springs Cave*; Transylvania Co.: Snake Den no. 2 Cave*; Yancey Co.: Celo Cave*, Isom Mica Mine*.

Conservation Status: IUCN: Near Threatened (Solari, 2018c); NatureServe: G1G2 (S2 in North Carolina); Listed as Threatened in North Carolina (North Carolina Wildlife Resources Commission, 2017a); Listed as a Species of Greatest Conservation Need in North Carolina (North Carolina Wildlife Resources Commission, 2015); Listed as Threatened under the U.S. Endangered Species Act.

Comments: Myotis septentrionalis is one of the bat species most impacted by White-nose

Syndrome. The species was listed as Threatened under the Endangered Species Act, effective 4 May 2015 (USFWS, 2015). It is considered uncommon in the mountainous areas of western North Carolina where significant declines have been documented (North Carolina Wildlife Resources Commission, 2017b; O'Keefe et al., 2019).

REFERENCES: ${ }^{1}$ Boynton et al. (1992); ${ }^{2}$ VertNet: MVZ; ${ }^{3}$ VertNet: NCSM; * this study.

\section{Myotis sodalis Miller \& Allen, 1928 (TX) Indiana Bat}

LocAlitIES: Jackson Co.: Kitchen Cave ${ }^{2,3}$; Madison Co.: Anthodite Cave'; McDowell Co.: Linville Caverns', Wind Cave'; Rutherford Co.: Bat Cave1-5; Swain Co.: Blowing Springs Cave1, Hewitt Station Mine², Lost Nantahala Cave1.

Conservation Status: IUCN: Near Threatened (Arroyo-Cabrales and Ospina-Garces, 2016); NatureServe: G2 (S1S2 in North Carolina); Listed as Endangered in North Carolina (NCWRC, 2017); Listed as a Species of Greatest Conservation Need in North Carolina (North Carolina Wildlife Resources Commission, 2015); Listed as Endangered under the U.S. Endangered Species Act.

Comments: Two caves and one mine in North Carolina are considered priority sites for this federally endangered bat (USFWS, 2007). Records from Madison, McDowell and Swain counties (other than Hewitt Station Mine) by Boynton et al. (1992) have not been confirmed and are based on questionable identifications. Like many other cave-hibernating bat species, populations of M. sodalis have suffered declines due to White-nose Syndrome. Myotis sodalis was never common in North Carolina hibernacula before the arrival of White-nose Syndrome but now are rarely encountered (Katherine Caldwell, North Carolina Wildlife Resources Commission, personal communication in O'Keefe et al., 2019). RefERENCES: ${ }^{1}$ Boynton et al. (1992); ${ }^{2}$ USFWS (2007); ${ }^{3}$ VertNet: MVZ; ${ }^{4}$ VertNet: NCSM; ${ }^{5}$ VertNet: UMMZ.

\section{Genus Perimyotis}

\section{Perimyotis subflavus (Cuvier, 1832) (TX) Tri-colored Bat}

Localities: Avery Co.: Black Rock Cliffs Cave², Cranberry Mines*; Jackson Co.: Kitchen Cave*; Madison Co.: Anthodite

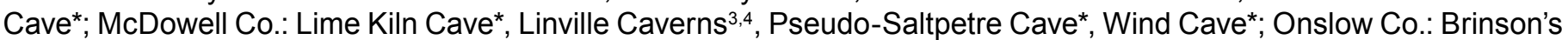
Rockhouse Cave ${ }^{2}$; Rutherford Co.: Bat Cave ${ }^{1,4,5}$; Breakdown Cave*, Halloween Haven*, Moonshiners Cave*, Rumbling Bald Cave*, Sliding Rock Cave*; Swain Co.: Blowing Springs Cave²; Transylvania Co.: Snake Den no. 2 Cave*; Yancey Co.: Celo Cave*, Cooper's Cave*, Isom Mica Mine*.

Conservation Status: IUCN: Vulnerable A3bce (Solari, 2018d); NatureServe: G2G3 (S3 in North Carolina); Listed as a Species of Greatest Conservation Need in North Carolina (North Carolina Wildlife Resources Commission, 2015). 
Comments: Perimyotis subflavus was quite common in caves of North Carolina before White-nose Syndrome spread into the region but have experienced significant declines in the Southern Appalachians (O'Keefe et al., 2019). Its conservation status was recently elevated to Vulnerable due to impacts from White-nose Syndrome. In addition, $P$. subflavus is under review by USFWS for potential listing.

RefERENCES: ${ }^{1}$ VertNet: MVZ; ${ }^{2}$ VertNet: NCSM; ${ }^{3}$ VertNet: ROM; ${ }^{4}$ VertNet: UMMZ; ${ }^{5}$ Bat Population Database; * this study.

\section{Order Didelphimorphia \\ Family Didelphidae \\ Genus Didelphis}

Didelphis virginiana (Kerr, 1792) (AC) Virginia Opossum

LocAlities: McDowell Co.: Holler's Potato Hole no. 2*; Rutherford Co.: Moonshiners Cave*, Rumbling Bald Cave*.

Conservation Status: IUCN: Least Concern; NatureServe: G5 (S5 in North Carolina).

COMmENTS: Opossums have been reported from caves in the eastern United States (Dearolf, 1956; Cliburn and Middleton, 1983; Niemiller, unpublished data), but caves are thought to be much less important to this species compared to Procyon lotor.

REFERENCES: * this study.

\section{Order Rodentia \\ Family Cricetidae \\ Genus Neotoma}

\section{Neotoma floridana haematoreia Howell, 1934 (TX) Southern Appalachian Woodrat}

Localities: McDowell Co.: Lime Kiln Cave*, Wind Cave*; Rutherford Co.: Moonshiners Cave*, Rumbling Bald Cave'; Yancey Co.: Celo Cave*.

Conservation Status: IUCN: Least Concern; NatureServe: G5T4Q (S3S4 in North Carolina); Listed as a Species of Greatest Conservation Need in North Carolina (North Carolina Wildlife Resources Commission, 2015).

CommenTs: Nests and scat are common in North Carolina caves. The IUCN Red List status rank reflects the conservation status of the species and not this subspecies.

RefERENCES: ${ }^{1}$ VertNet: NCSM; * this study.

\section{Family Dipodidae \\ Genus Napaeozapus}

Napaeozapus insignis (Miller, 1891) (TX/AC) Woodland Jumping Mouse

LOCALITIES: Henderson Co.: Middle Fork Shelter Cave no. $2^{*}$.

Conservation Status: IUCN: Least Concern; NatureServe: G5 (S4 in North Carolina).

COMmENTS: Mice are not uncommon in caves, although few records for this particular species are known.

REFERENCES: * this study.

\section{Summary of bioinventory and literature records}

We compiled occurrence records from 127 caves and other subterranean habitats in 29 counties in North Carolina from the literature and biological surveys. In total, this dataset includes occurrences for at least 5 phyla, 17 classes, 43 orders, 90 families, 124 genera, and 164 species (Table 1; Figure 2). Twenty-nine taxa are aquatic, whereas 135 taxa are terrestrial. Diverse invertebrate groups included spiders (Order Araneae; 11 families, 18 genera, 22 species), springtails (Class Collembola; seven families, nine genera, 15 species), copepods (Class Maxillopoda; one family, four genera, seven species), clitellate worms (Class Clitellata; three families, eight genera, eight species), and snails (Class Gatropoda; six families, nine genera, 12 species. The most common invertebrate species included the Cave Orbweaver (Meta ovalis) (21 occurrences from eight counties), a cave-obligate dipluran (Litocampa sp.) (15 occurrences from six counties), a cave cobweb spider (Nesticus brimleyi) (11 occurrences from two counties), and an undescribed species of Stygobromus amphipod (11 occurrences from five counties). Thirty-two vertebrate taxa were documented, including four fishes, nine salamanders, one lizard, four snakes, two birds, and 12 mammals. Mammal diversity was dominated by bats (eight species), with Tri-colored bats (Perimyotis subflavus) documented most frequently (20 occurrences). Most of the vertebrates reported to date in North Carolina have been previously documented from caves in adjacent states (e.g., Buhlmann, 2001; Fong et al., 2012; Niemiller et al., 2016).

At least 25 taxa are considered cave obligates, i.e., troglobites and stygobites (Table 2), with the greatest troglobitic/ stygobitic species richness observed in Phagocata flatworms (five species), Pseudosinella springtails (five species), and Stygobromus amphipods (three described species and at least two undescribed taxa). Three of the 25 cave-ob- 
Table 1. Taxonomic diversity of invertebrates and vertebrates from caves and other subterranean habitats in North Carolina.

\begin{tabular}{|c|c|c|c|c|c|}
\hline Taxon & No. of Orders & No. of Families & No. of Genera & No. of Taxa & No. of Troglobites \\
\hline \multicolumn{6}{|l|}{ Phylum Platyhelminthes } \\
\hline Class Turbellaria & 1 & 1 & 1 & 6 & 5 \\
\hline \multicolumn{6}{|l|}{ Phylum Annelida } \\
\hline Class Clitellata & 1 & 3 & 8 & 8 & 0 \\
\hline \multicolumn{6}{|l|}{ Phylum Arthropoda } \\
\hline Class Arachnida & 6 & 23 & 30 & 34 & 3 \\
\hline Class Chilopoda & 2 & 2 & 2 & 2 & 0 \\
\hline Class Collembola & 3 & 7 & 9 & 17 & 5 \\
\hline Class Diplopoda & 4 & 6 & 6 & 8 & 2 \\
\hline Class Entognatha & 1 & 2 & 2 & 2 & 1 \\
\hline Class Insecta & 9 & 19 & 21 & 22 & 0 \\
\hline Class Malacostraca & 3 & 5 & 6 & 13 & 8 \\
\hline Class Maxillopoda & 1 & 1 & 4 & 7 & 1 \\
\hline Class Symphyla & 1 & 1 & 1 & 1 & 0 \\
\hline \multicolumn{6}{|l|}{ Phylum Mollusca } \\
\hline Class Gastropoda & 2 & 6 & 9 & 12 & 0 \\
\hline \multicolumn{6}{|l|}{ Phylum Chordata } \\
\hline Class Actinopterygii & 2 & 2 & 4 & 4 & 0 \\
\hline Class Amphibia & 1 & 1 & 6 & 9 & 0 \\
\hline Class Aves & 1 & 2 & 2 & 2 & 0 \\
\hline Class Mammalia & 4 & 5 & 8 & 12 & 0 \\
\hline Class Reptilia & 1 & 4 & 5 & 5 & 0 \\
\hline Total & 43 & 90 & 124 & 164 & 25 \\
\hline
\end{tabular}

ligate taxa (Phanetta subterranea, Porrhomma cavernicola, and Pseudosinella pecki) have broad distributions that include several karst regions in the eastern United States. The other cave-obligate taxa are predominately endemic to North Carolina. No cave-obligate vertebrates have been documented from North Carolina to date.

Of the taxa included in the checklist above, at least 25 taxa are considered troglophiles/stygophiles, 50 taxa as trogloxenes/stygoxenes, and 22 as accidentals. All taxa classified as accidentals are considered common in surface habitats, except for Mesodon andrewsae. This terrestrial snail is considered Vulnerable (G3) globally and Imperiled-Vulnerable (S2S3) in North Carolina. All eight earthworms reported from North Carolina caves (Reynolds, 1994) are considered edaphic. The ecological classification of 44 taxa could not confidently be assigned to one specific category: seven taxa were classified as troglophiles or trogloxenes (stygophiles or stygoxenes) and 27 taxa as trogloxenes or accidentals.

Counties with the greatest number of caves with biological occurrences include Rutherford, McDowell, Swain, Henderson, Polk, and Avery counties (Table 3). Unsurprisingly, these counties also have the greatest subterranean species richness in North Carolina. However, most occurrences for each of these counties are for just a few cave systems. Twenty or more taxa have been documented at four caves (Table 4): Bat Cave complex in Rutherford County (34 taxa), Blowing Springs Cave in Swain County (27 taxa), Linville Caverns in McDowell County (27 taxa), and Rumbling Bald Cave in Rutherford County (22 taxa).

Caves with the greatest troglobitic species richness include the Bat Cave complex in Rutherford County, Blowing Springs Cave in Swain County, and Wind Cave in McDowell County, each with four troglobites documented. A particularly rich vertebrate fauna is known from Linville Caverns with 14 taxa, including four fishes, six salamanders, and four bat species. A diverse vertebrate fauna ( $>5$ taxa) also has been documented to date at Rumbling Bald Cave in 


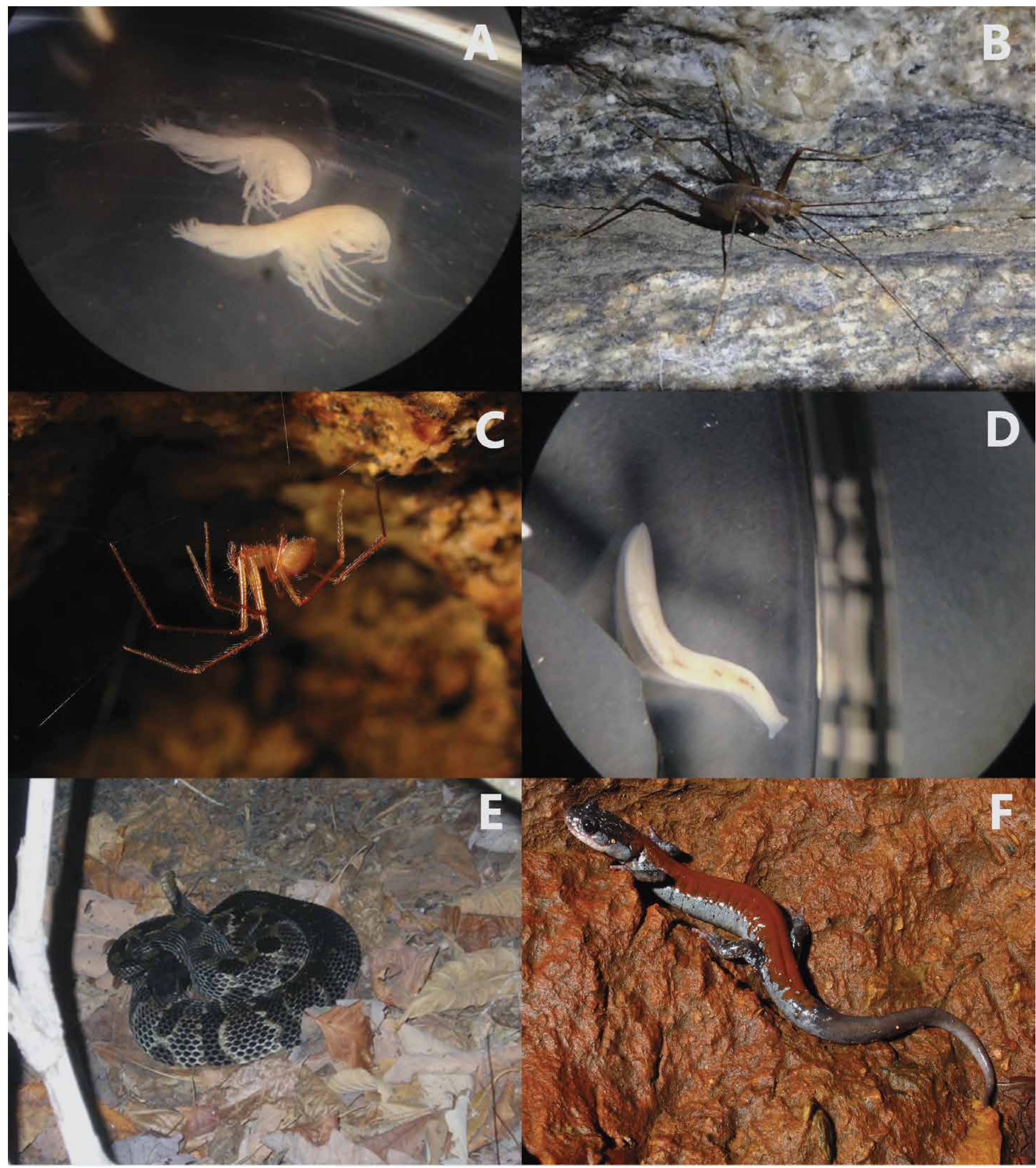

Figure 2. Representative cave life documented in North Carolina caves: A) Stygobromus sp. from Wind Cave, McDowell County (Photo by Cato Holler); B) Euhadenoecus adelphus from Bat Cave, Rutherford County (Photo by Cato Holler) C) Nesticus sp. from Wind Cave, McDowell County (Photo by Alan Cressler); D) Phagocata sp. from Wind Cave, McDowell County (Photo by Cato Holler); E) Crotalus horridus from Crotalus Shelter, McDowell County (Photo by Cato Holler); F) Plethodon yonahlossee from Wind Cave, McDowell County (Photo by Alan Cressler). 
Table 2. Troglobitic and stygobitic taxa documented from caves and other subterranean habitats in North Carolina.

\begin{tabular}{|c|c|c|c|}
\hline Species & Authority & $\begin{array}{c}\text { No. of } \\
\text { Occurrences }\end{array}$ & Counties \\
\hline Caecidotea carolinensis & Lewis \& Bowman, 1977 & 1 & McDowell \\
\hline Diacyclops jeanneli putei & (Yeatman, 1943) & 1 & Orange \\
\hline Litocampa sp. & & 15 & $\begin{array}{l}\text { Henderson, Madison, McDowell, Polk, Rutherford, } \\
\text { Swain }\end{array}$ \\
\hline Nannopetalum vespertilio & Shear, 2003 & 1 & Rutherford \\
\hline Nesticus brimleyi & Gertsch, 1984 & 11 & Polk, Rutherford \\
\hline Phagocata carolinensis & Kenk, 1979 & 2 & Burke, McDowell \\
\hline Phagocata holleri & Kenk, 1979 & 1 & Ashe \\
\hline Phagocata procera & Kenk, 1984 & 7 & Burke, Jackson, McDowell, Mitchell, Yancey \\
\hline Phagocata pygmaea & Kenk, 1987 & 1 & Stokes \\
\hline Phagocata spuria & Kenk, 1987 & 2 & McDowell \\
\hline Phanetta subterranea & (Emerton, 1875) & 4 & McDowell, Rutherford, Yancey \\
\hline Porrhomma cavernicola & (Keyserling, 1886) & 2 & Swain \\
\hline Pseudosinella flatua & Christiansen \& Bellinger, 1996 & 1 & Swain \\
\hline Pseudosinella gisini carolina & Christiansen \& Bellinger, 1996 & 2 & Rutherford \\
\hline Pseudosinella orba & Christiansen, 1961 & 1 & Polk \\
\hline Pseudosinella pecki & Christiansen \& Bellinger, 1980 & 1 & Swain \\
\hline Pseudosinella vespera & Christiansen \& Bellinger, 1996 & 2 & Rutherford, Swain \\
\hline Pseudotremia shelleyi & Lewis, 2009 & 4 & McDowell, Rutherford \\
\hline Stygobromus araeus & (Holsinger, 1969) & 1 & Gates \\
\hline Stygobromus carolinensis & Holsinger, 1978 & 2 & Yancey \\
\hline Stygobromus cf. carolinensis & & 1 & Wilkes \\
\hline Stygobromus indentatus & (Holsinger, 1967) & 1 & Nash \\
\hline Stygobromus nov. sp. A & & 11 & Avery, Burke, Henderson, McDowell, Mitchell \\
\hline Stygobromus nov. sp. B & & 1 & Jackson \\
\hline Stygobromus sp. & & 4 & Buncombe, McDowell, Polk \\
\hline
\end{tabular}

Rutherford County (8 taxa), Bat Cave complex in Rutherford County (6 taxa), Wind Cave in McDowell County (7 taxa), and Cooper's Cave in Yancey County (6 taxa) (Table 4).

\section{Species of conservation concern}

Several species documented from caves and other subterranean habitats in North Carolina are of conservation concern, including 14 species that are troglobites or stygobites (Table 5). Five species are on the IUCN Red List of Threatened Species. The bats Myotis lucifugus and M. leibii have been evaluated as Endangered and the Tri-colored Bat (Perimyotis subflavus) and groundwater amphipods Stygobromus araeus and S. indentatus have been evaluated as Vulnerable. Two additional bats, M. septentrionalis and M. sodalis, and the Hickory Nut Gorge Green Salamander (Aneides caryaensis) have been evaluated as Near Threatened. Aneides caryaensis likely will be placed on the IUCN Red List in the near future. The conservation status of four bat species on the IUCN Red List was recently increased in response to population declines associated with White-nose Syndrome (Solaris 2018a,b,c,d).

Based on NatureServe conservation rank criteria, 21 species are of conservation concern at the global level (G1G3), many of which are troglobites or stygobites. Nesticus cooperi, Stygobromus carolinensis, N. carolinensis, and Helicodiscus saludensis are ranked as Critically Imperiled (G1). In addition, three species are ranked as Imperiled (G2), five species as Vulnerable (G3), six species as Critically Imperiled-Imperiled (G1G2), and three species as Imperiled-Vulnerable (G2G3). At the state level, 28 species of conservation concern, including two species ranked as Possibly Extinct (SH), seven species as Critically Imperiled (S1), four species as Imperiled (S2), five as Vulnerable (S3), seven species as Critically Imperiled-Imperiled (S1S2), and three species as Imperiled-Vulnerable (S2S3).

Nine species are listed as protected species in North Carolina by the North Carolina Wildlife Resources Commission (North Carolina Wildlife Resources Commission, 2017a; Table 5), including three species as Endangered, three species as Threatened, and three species as Special Concern. Three bats are also listed under the U.S. Endangered 
Table 3. Subterranean occurrences and taxa by county and caves sampled in North Carolina. Percent is the number of caves with records divided by the total number of caves multiplied by 100 in a county. Some counties include sites that are not caves and are not included in this calculation.

\begin{tabular}{|c|c|c|c|c|c|}
\hline County & No. of Records & No. of Taxa & $\begin{array}{l}\text { No. of Sites with } \\
\text { Records }\end{array}$ & $\begin{array}{l}\text { Total No. of } \\
\text { Caves }\end{array}$ & $\begin{array}{c}\text { Percent } \\
(\%)\end{array}$ \\
\hline Alexander & 0 & 0 & 0 & 11 & 0.0 \\
\hline Alleghany & 0 & 0 & 0 & 10 & 0.0 \\
\hline Anson & 0 & 0 & 0 & 9 & 0.0 \\
\hline Ashe & 3 & 2 & 2 & 32 & 6.3 \\
\hline Avery & 27 & 14 & 12 & 136 & 8.1 \\
\hline Bladen & 0 & 0 & 0 & 2 & 0.0 \\
\hline Buncombe & 2 & 2 & 1 & 41 & 2.4 \\
\hline Burke & 11 & 7 & 11 & 254 & 4.3 \\
\hline Caldwell & 4 & 4 & 2 & 7 & 28.6 \\
\hline Chatham & 7 & 4 & 6 & 1 & 0.0 \\
\hline Cherokee & 0 & 0 & 0 & 8 & 0.0 \\
\hline Clay & 0 & 0 & 0 & 17 & 0.0 \\
\hline Columbus & 0 & 0 & 0 & 10 & 0.0 \\
\hline Craven & 0 & 0 & 0 & 1 & 0.0 \\
\hline Dare & 0 & 0 & 0 & 1 & 0.0 \\
\hline Davidson & 3 & 3 & 2 & 8 & 25.0 \\
\hline Davie & 0 & 0 & 0 & 1 & 0.0 \\
\hline Duplin & 0 & 0 & 0 & 3 & 0.0 \\
\hline Forsyth & 0 & 0 & 0 & 1 & 0.0 \\
\hline Gaston & 0 & 0 & 0 & 20 & 0.0 \\
\hline Gates & 1 & 1 & 1 & 0 & $\cdots$ \\
\hline Harnett & 0 & 0 & 0 & 1 & 0.0 \\
\hline Haywood & 0 & 0 & 0 & 38 & 0.0 \\
\hline Henderson & 30 & 27 & 7 & 46 & 15.2 \\
\hline Hoke & 0 & 0 & 0 & 4 & 0.0 \\
\hline Iredell & 0 & 0 & 0 & 6 & 0.0 \\
\hline Jackson & 9 & 9 & 4 & 23 & 13.0 \\
\hline Jones & 1 & 1 & 1 & 4 & 0.0 \\
\hline Lincoln & 0 & 0 & 0 & 1 & 0.0 \\
\hline Macon & 12 & 10 & 3 & 24 & 12.5 \\
\hline Madison & 15 & 12 & 5 & 28 & 17.9 \\
\hline Martin & 0 & 0 & 0 & 2 & 0.0 \\
\hline Mecklenburg & 0 & 0 & 0 & 2 & 0.0 \\
\hline McDowell & 82 & 54 & 11 & 259 & 4.2 \\
\hline Mitchell & 5 & 3 & 3 & 12 & 8.3 \\
\hline Montgomery & 0 & 0 & 0 & 9 & 0.0 \\
\hline Nash & 1 & 1 & 1 & 0 & $\ldots$ \\
\hline New Hanover & 0 & 0 & 0 & 4 & 0.0 \\
\hline Northhampton & 0 & 0 & 0 & 5 & 0.0 \\
\hline Onslow & 4 & 4 & 2 & 5 & 20.0 \\
\hline Orange & 2 & 2 & 1 & 11 & 0.0 \\
\hline Polk & 30 & 25 & 8 & 17 & 47.1 \\
\hline Randolph & 0 & 0 & 0 & 13 & 0.0 \\
\hline Rockingham & 2 & 2 & 1 & 1 & 100.0 \\
\hline Rowan & 0 & 0 & 0 & 2 & 0.0 \\
\hline Rutherford & 137 & 76 & 20 & 186 & 10.8 \\
\hline Stokes & 2 & 2 & 2 & 96 & 2.1 \\
\hline Surry & 1 & 1 & 1 & 17 & 5.9 \\
\hline Swain & 47 & 35 & 9 & 40 & 22.5 \\
\hline Transylvania & 7 & 7 & 1 & 35 & 2.9 \\
\hline Union & 0 & 0 & 0 & 2 & 0.0 \\
\hline Wake & 1 & 1 & 1 & 12 & 8.3 \\
\hline Watauga & 2 & 2 & 2 & 44 & 4.5 \\
\hline Wilkes & 2 & 2 & 2 & 30 & 6.7 \\
\hline Yancey & 25 & 17 & 5 & 31 & 9.7 \\
\hline Total & 475 & 164 & 127 & 1,582 & 8.0 \\
\hline
\end{tabular}


Table 4. List of the 14 most diverse cave systems in North Carolina.

\begin{tabular}{|c|c|c|c|c|c|}
\hline Cave & County & No. of Taxa & $\begin{array}{c}\text { No. of } \\
\text { Troglobites }\end{array}$ & $\begin{array}{c}\text { No. of } \\
\text { Vertebrates }\end{array}$ & $\begin{array}{c}\text { No. of Species of } \\
\text { Conservation Concern }\end{array}$ \\
\hline Bat Cave complex & Rutherford & 34 & 4 & 6 & 8 \\
\hline Blowing Springs Cave & Swain & 27 & 4 & 5 & 8 \\
\hline Linville Caverns & McDowell & 27 & 1 & 14 & 4 \\
\hline Rumbling Bald Cave & Rutherford & 22 & 3 & 8 & 7 \\
\hline Staircase Cave & McDowell & 16 & 3 & 1 & 1 \\
\hline Wind Cave & McDowell & 15 & 4 & 7 & 6 \\
\hline Breakdown Cave & Rutherford & 13 & 2 & 4 & 4 \\
\hline Devil's Kitchen Cave & Henderson & 12 & 0 & 0 & 0 \\
\hline Little Warrior Mountain Cave & Polk & 11 & 3 & 1 & 2 \\
\hline Lost Nantahala Cave & Swain & 11 & 2 & 2 & 4 \\
\hline Western Talus Tunnel & Rutherford & 10 & 0 & 0 & 0 \\
\hline Sliding Rock Cave & Rutherford & 10 & 1 & 3 & 4 \\
\hline Moonshiners Cave & Rutherford & 9 & 1 & 4 & 4 \\
\hline Celo Cave & Yancey & 9 & 0 & 4 & 4 \\
\hline
\end{tabular}

Table 5. List of species of conservation concern and their conservation status documented from caves and other subterranean habitats in North Carolina. Conservation status includes IUCN Red List ranking, NatureServe global conservation rank, NatureServe state conservation rank, state designation by NCWRC, and federal status under the U.S. Endangered Species Act. Species considered troglobites or stygobites are in bold.

\begin{tabular}{|c|c|c|c|c|c|c|}
\hline Scientific name & Common name & IUCN & NS Global & NS State & $\begin{array}{c}\text { NC } \\
\text { Status }\end{array}$ & $\begin{array}{c}\text { US } \\
\text { Status }\end{array}$ \\
\hline Phagocata carolinensis & Carolina Cave Planarian & & G1G2 & S1S2 & & \\
\hline Phagocata holleri & Holler's Cave Planarian & & G1G2 & S1S2 & & \\
\hline Phagocata procera & A Cave Planarian & & G1G2 & S1S2 & & \\
\hline Hypochilus coylei & A Lampshade Weaver & & G3? & S3? & & \\
\hline Nesticus brimleyi & A Cave Cobweb Spider & & G1G2 & S1? & & \\
\hline Nesticus carolinensis & Linville Caverns Spider & & G1? & S1 & & \\
\hline Nesticus cooperi & Lost Nantahala Cave Spider & & $\mathrm{G} 1$ & $\mathrm{~S} 1$ & & \\
\hline Nesticus mimus & A Cobweb Spider & & $\mathrm{G} 2$ & $\mathrm{~S} 2 ?$ & & \\
\hline Pseudosinella flatua & Blowing Springs Cave Springtail & & G1G2 & S1S2 & & \\
\hline Pseudosinella gisini carolina & Carolina Cave Springtail & & G3G4T1T2 & S1S2 & & \\
\hline Pseudosinella pecki & Peck's Cave Springtail & & G2G3 & SNR & & \\
\hline Pseudosinella vespera & Bat Cave Springtail & & G2 & S1S2 & & \\
\hline Stygobromus araeus & Tidewater Interstitial Amphipod & VU & G3 & SU & & \\
\hline Stygobromus carolinensis & Carolina Groundwater Amphipod & & G1 & s1 & & \\
\hline Stygobromus indentatus & Tidewater Amphipod & VU & G3 & SU & & \\
\hline Stygobromus nov. sp. B & A Cave Amphipod & & GNR & S1? & & \\
\hline Caecidotea carolinensis & Bennett's Mill Cave Isopod & & G2G3 & SH & LE & \\
\hline Diacyclops jeanneli putei & Carolina Well Copepod & & G3G4 & SH & SC & \\
\hline Helicodiscus saludensis & Corncob Snail & & G1 & $\mathrm{S} 1 ?$ & & \\
\hline Mesodon andrewsae & Balsam Globe & & G3 & S2S3 & & \\
\hline Mesomphix latior & Broad Button & & G3G4 & S2S3 & & \\
\hline Aneides caryaensis & Hickory Nut Green Salamander & NT & G3G4 & S2S3 & LT & \\
\hline Corynorhinus rafinesquii & Rafinesque's Big-eared Bat & LC & G3G4T2 & S1 & LE & LE \\
\hline Corynorhinus townsendii virginianus & Virginia Big-eared Bat & LC & G3G4 & S3 & LT & \\
\hline Myotis leibii & Eastern Small-footed Bat & EN & G4 & $\mathrm{S} 2$ & SC & \\
\hline Myotis lucifugus & Little Brown Bat & EN & G3 & S3 & & \\
\hline Myotis septentrionalis & Northern Long-eared Bat & NT & G1G2 & S2 & LT & LT \\
\hline Myotis sodalis & Indiana Bat & NT & G2 & S1S2 & LE & LE \\
\hline Perimyotis subflavus & Tri-colored Bat & VU & G2G3 & S3 & & \\
\hline Crotalus horridus & Timber Rattlesnake & LC & G4 & S3 & SC & \\
\hline
\end{tabular}


Species Act, including Myotis sodalis and Corynorhinus townsendii virginianus as Endangered and M. septentrionalis as Threatened. Since White-nose Syndrome was first detected in North Carolina at a cave in Avery County in 2011, it has spread into 12 counties in western North Carolina (https://www.whitenosesyndrome.org/static-page/where-iswns-now) resulting in significant population declines for several bat species. Most impacted have been populations of M. septentrionalis, M. lucifugus, M. sodalis, and $P$. subflavus, in which some populations have experienced over 90 percent declines (North Carolina Wildlife Resources Commission, 2017b). Myotis septentrionalis was recently listed as Threatened on the U.S. Endangered Species Act in 2015 due to population declines associated with White-nose Syndrome (USFWS, 2015). Myotis lucifugus and $P$. subflavus may be listed in the near future; their respective conservation statuses (both IUCN Red List and NatureServe) were elevated to higher risk of extinction ranks recently due to high mortality from White-nose Syndrome (e.g., Frick et al., 2010; Verant et al., 2012).

\section{Conclusions and Future Directions}

Although not nearly as extensive and common as in adjacent states, such as Georgia, Tennessee, and Virginia, caves and other subterranean habitats in North Carolina support a diverse community of invertebrates and vertebrates. Our review of the subterranean fauna of North Carolina includes at least 164 taxa representing five phyla and 90 families. While this review highlights the biodiversity found in subterranean habitats in the state, we aim for this assessment to serve as a base line for future biological surveys in caves and other subterranean habitats in the state. Moreover, we highlight the importance of caves and subterranean habitats for many species, not just bats and troglobites (obligate cave species).

A small percentage of caves in North Carolina have biological occurrence records (8.0\%), and even a smaller fraction of sites has had comprehensive or repeated biological inventories conducted. Several counties, such as Buncombe, Burke, Haywood, Stokes, Transylvania, and Watauga counties, have $>30$ caves yet $<4 \%$ of caves have even a single biological occurrence. This biodiversity knowledge shortfall is not limited to North Carolina, but even occurs in states with considerably greater karst and cave systems (e.g., Tennessee; Niemiller and Zigler, 2013). Thus, there is great potential to document new cave distributional records. With 20 described cave-obligate taxa, North Carolina is tied for $19^{\text {th }}$ (with Pennsylvania) in cave-obligate species richness among U.S. states (Niemiller et al. 2019). Much of the cave-obligate diversity in North Carolina is endemic to the state. At least three undescribed taxa are noted in the literature and await formal taxonomic description. However, there is considerable potential to discover new taxa unknown to science in subterranean habitats of North Carolina, given the complex geological history, topography, and isolated nature of cave systems in much of the state.

Caves are important habitats for many species of vertebrates and invertebrates. Several caves and mines have been identified previously as important hibernacula for several threatened and endangered bat species in North Carolina (Boynton et al., 1992), and there is a growing body of literature that caves are critically important habitats for other vertebrates and invertebrates (e.g., Niemiller and Miller, 2009; Niemiller et al., 2016) for shelter, hibernation, food, and other aspects of life history.

\section{Acknowledgements}

We thank the following individuals for assistance with arranging access to caves and with bioinventories: the owners and management of Linville Caverns Incorporated, Chimney Rock, and Grandfather Mountain (the latter two are now state parks), along with the numerous private cave owners throughout the state. We also thank all past and present members of the Flittermouse Grotto. Peter Hertl did a considerable amount of collecting in the early days of the project. Bob Currie, Allen Boynton, Chris McGrath and other members of the U.S. Fish and Wildlife and N.C. Wildlife Resources Commission were kind to allow us to accompany them on many of their official bat inventories. Lead author $(\mathrm{CH})$ is particularly grateful to his wife, Susan Holler, and three children, Oliver, Chris, and Nancy, for their encouragement and assistance in the field over many years. Thank you Alan Cressler for providing photographs. We especially thank the late Dr. John Cooper, former Director of Research and Collections at the North Carolina State Museum, who first inspired and encouraged cavers to initiate a cave fauna survey in the state. John was also instrumental in distributing many of the collected specimens to taxonomic specialists for identification. We also thank the following taxonomists for their assistance with this project: Tom Barr, Ken Christiansen, Fredrick Coyle, Lynn Ferguson, Marshal Hedin, John Holsinger, Roman Kenk, William Shear, Rowland Shelly, and Charles Stephen.

\section{Literature Cited}

Arroyo-Cabrales, J., and Ospina-Garces, S., 2016, Myotis sodalis: The IUCN Red List of Threatened Species 2016: e.T14136A22053184. http:// dx.doi.org/10.2305/IUCN.UK.2016-1.RLTS.T14136A22053184.en. Downloaded on 04 January 2019.

Barr, T.C., Jr., 1968, Cave ecology and the evolution of troglobites: in Dobzhansky, T., Hecht, M.K., and Steere, W.C., eds., Evolutionary Biology, vol. 2: New York, Appleton-Century-crofts, p. 35-102.

Beane, J.C., Braswell, A.L., Mitchell, J.C., Palmer, W.M., and Harrison III, J.R., 2010, Amphibians and Reptiles of the Carolinas and Virginia, $2^{\text {nd }}$ edition: Chapel Hill, The University of North Carolina Press, 274 p. 
Boynton A., Holler, C., and Currie, B., 1992, Survey of important sites for listed and candidate bat species in western North Carolina: Final report to the U.S. Fish and Wildlife Service, 13 .

Brandon, R.A., 1966, Systematics of the Salamander Genus Gyrinophilus: Urbana, University of Illinois Press, Illinois Biological Monographs 35, $82 \mathrm{p}$.

Brode, W.E., 1958, The occurrence of the pickerel frog, three salamanders and two snakes in Mississippi caves: Copeia, v. 1958, p. 47-48.

Bruno, M.C., Reid, J.W., and Perry, S.A., 2000, New records of copepods from Everglades National Park (Florida): description of two new species of Elaphoidella (Harpacticoida, Canthocamptidae), and supplementary description of Diacyclops nearcticus (Cyclopoida, Cyclopidae): Crustaceana, v. 73, p. 1171-1204.

Buhlmann, K.A., 2001, A biological inventory of eight caves in northwestern Georgia with conservation implications: Journal of Cave and Karst Studies, v. 63, p. 91-98.

Camp, C.D., and Jensen, J.B., 2007, Use of twilight zones of caves by plethodontid salamanders: Copeia, v. 2007, p. 594-604.

Christ, C.A., 2003, The invertebrate community of the Rumbling Bald Mountain Fissure Cave System: Unpublished report, Warren Wilson College, Ashville, North Carolina, $28 \mathrm{p}$.

Christiansen, K., and Bellinger, P., 1980, The Collembola of North America North of the Rio Grande: Grinnell College, Grinnell, lowa, 1322 p.

Christiansen, K., and Bellinger, P., 1996, Cave Pseudosinella and Oncopodura new to science: Journal of Cave and Karst Studies, v. 58, p. 38-53.

Cliburn, J.W., and Middleton, Jr., A.L., 1983, The vertebrate fauna of Mississippi caves: Bulletin of the National Speleological Society, v. 45, p. 45-48.

Cooper, J.E., and Cooper, M.R., 1968, Cave-associated herpetozoa II: salamanders of the genus Gyrinophilus in Alabama caves: Bulletin of the National Speleological Society, v. 30, p. 19-24.

Culver, D. C., Christman, M. C., Elliott, W. R., Hobbs, H. H., and Reddell, J. R., 2003, The North American obligate cave fauna: regional patterns: Biodiversity \& Conservation, v. 12, p. 441-468.

Culver, D.C., and Pipan, T., 2009, The Biology of Caves and Other Subterranean Habitats: Oxford University Press, 254 p.

Culver, D.C., Holsinger, J.R., and Feller, D.J., 2012, The fauna of seepage springs and other shallow subterranean habitats in the Mid-Atlantic Piedmont and Coastal Plain: Northeastern Naturalist, v. 19, p. 1-42.

Dearolf, K., 1956, Survey of North American cave vertebrates: Proceedings of the Pennsylvania Academy of Science, v. 30, p. $201-210$.

Dodd, C.K. Jr., Griffey, M.L., and Corser, J.D., 2001, The cave associated amphibians of Great Smoky Mountains National Park: Review and monitoring: Journal of the Elisha Mitchell Scientific Society, v. 117, p. 139-149.

Dourson, D.C., 2013, Land Snails of the Great Smoky Mountains National Park and Southern Appalachians: Goatslug Publications, Bakersville, North Carolina, $336 \mathrm{p}$.

Ellison, L., O'Shea, T., Bogan, M., Everette, A., and Schneider, D., 2003, Existing data on colonies of bats in the United States: Summary and analysis of the US Geological Survey's bat population database: USGS/BRD/ITR-2003-0003, Fort Collins, Colorado, US Geological Survey.

Faber-Langendoen, D., Nichols, J., Master, L., Snow, K., Tomaino, A., Bittman, R., Hammerson, G., Heidel, B., Ramsay, L., Teucher, A., and Young, B., 2012, NatureServe Conservation Status Assessments: Methodology for Assigning Ranks, revised edition: Arlington, Virginia, NatureServe, $44 \mathrm{p}$.

Flock, B., 2013, 2013 Tennessee Bat Population Monitoring and White Nose Syndrome: Nashville, Tennessee Wildlife Resources Agency, TWRA Wildlife Technical Report 13-22, 17 p.

Flock, B., 2014, 2014 Bat Population Monitoring and White Nose Syndrome Surveillance: Nashville, Tennessee Wildlife Resources Agency, TWRA Wildlife Technical Report 14-07, 15 p.

Fong, D.W., Porter, M.L., and Slay, M.E., 2012, Cave Life of the Virginias: A Field Guide to Commonly Encountered Species: Huntsville, Alabama, Biology Section of the National Speleological Society. $41 \mathrm{p}$.

Frick, W.F., Pollock, J.F., Hicks, A.C., Langwig, K.E., Reynolds, D.S., Turner, G.G., Butchkoski, C.M., and Kunz, T.H., 2010, An emerging disease causes regional population collapse of a common North American bat species: Science, v. 329, p. 679-682. doi:10.1126/ science.1188594.

Gaddy, L.L., 1986a, Natural Area Reconnaissance: Little Warrior Mountain Cave: Unpublished report. 4 p.

Gaddy, L.L., 1986b, The spider fauna of three caves in the Southern Appalachians of North Carolina: Unpublished report. 7 p.

Garton, E.R., Grady, F., and Carey, S.D., 1993, The Vertebrate Fauna of West Virginia Caves: West Virginia Speleological Survey, Bulletin 11, $107 \mathrm{p}$.

Gertsch, W.J., 1984, The spider family Nesticidae (Araneae) in North America, Central America, and the West Indies: Texas Memorial Museum Bulletin, v. 31, p. 1-91.

Godwin, J.C., 2008, Biological Inventory of the Cave and Karst Systems of The Nature Conservancy's Sharp-Bingham Mountain Preserve: Auburn, Alabama, Alabama Natural Heritage Program, $40 \mathrm{p}$.

Hammerson, G., 2004, Aneides aeneus. The IUCN Red List of Threatened Species 2004: e.T1282A3385547. http://dx.doi.org/10.2305/IUCN. UK.2004.RLTS.T1282A3385547.en. Downloaded on 04 January 2019.

Hedin, M.C., 1997a, Molecular phylogenetics at the population/species interface in cave spiders of the southern Appalachians (Araneae: Nesticidae: Nesticus): Molecular Biology and Evolution, v. 14, p. 309-324.

Hedin, M.C., 1997b, Speciational history in a diverse clade of habitat-specialized spiders (Araneae: Nesticidae: Nesticus): inferences from geographic-based sampling: Evolution, v. 51, p. 1929-1945.

Hertl, P.T., 1981, A preliminary inventory survey and management plan for the Bat Cave complex, Bat Cave, North Carolina: Unpublished report for the North Carolina Nature Conservancy.

Highton, R., and Peabody, R.B., 2000, Geographic protein variation and speciation in salamanders of the Plethodon jordani and Plethodon glutinosus complexes in the southern Appalachian Mountains with the description of four new species: in Bruce, R.C., Jaeger, R.G., and Houck, L.D., eds. The Biology of Plethodontid Salamanders: New York, Kluwer Academic / Plenum Publishers, p. 31-93.

Hobbs III, H.H., 2012, Diversity patterns in the United States: in White, W.B., Culver, D.C., eds. Encyclopedia of Caves, 2nd edition: Amsterdam, Academic Press, p. 251-264.

Hoffman, R.L., 1981, On the taxonomic status, distribution, and subspecies of the milliped Pseudotremia fracta Chamberlin (Chordeumatida, Cleidogonidae): Brimleyana, v, 5, p. 135-144.

Holland, D.G., 1981, A survey of the reptiles and amphibians of Bat Cave: Report to the North Carolina Nature Conservancy. 17 p.

Holler, C.O., 1981, North Carolina's Bat caves: a significant region of tectonokarst: in Proceedings of the $8^{\text {th }}$ International Congress of Speleology, Volume 1, Bowling Green, Kentucky, p. 190-191. 
Holler, C.O., 2019, Pseudokarst: in White, W.B., Culver, D.C., and Pipan, T., eds. Encyclopedia of Caves, $3^{\text {rd }}$ edition: Amsterdam, Academic Press, p. 836-849.

Holler, C.O., and Holler, S.G., 2009, Tectonic pseudokarst in western North Carolina: Field Trip Guidebook for the $15^{\text {th }}$ International Congress of Speleology, National Speleological Society, Huntsville, Alabama, $36 \mathrm{p}$.

Holsinger, J.R., 1978, Systematics of the subterranean amphipod genus Stygobromus (Crangonyctidae), Part II: Species of the eastern United States: Smithsonian Contributions to Zoology, v. 266, p. 1-144.

Holsinger, J.R., and Peck, S.B., 1971, The invertebrate cave fauna of Georgia: National Speleological Society Bulletin, v. 33, p. 23-44.

Hubbell, T.H., and Norton, R.M., 1978, The systematics and biology of the cave-crickets of the North American tribe Hadenoecini (Orthoptera Saltatoria: Ensifera: Rhaphidophoridae: Dolichopodinae): Miscellaneous Publications Museum of Zoology, Univ. of Michigan, No. 156, 124 p.

Inland Water Crustacean Specialist Group, 1996a, Stygobromus araeus: The IUCN Red List of Threatened Species 1996: e.T20973A9240768. http://dx.doi.org/10.2305/IUCN.UK.1996.RLTS.T20973A9240768.en. Downloaded on 04 January 2019.

Inland Water Crustacean Specialist Group, 1996b, Stygobromus identatus: The IUCN Red List of Threatened Species 1996: e.T20993A9242203. http://dx.doi.org/10.2305/IUCN.UK.1996.RLTS.T20993A9242203.en. Downloaded on 04 January 2019.

International Union for the Conservation of Nature, 2012, IUCN Red List Categories and Criteria: Version 3.1. Second edition: Gland, Switzerland, and Cambridge, UK, IUCN, $32 \mathrm{p}$.

Kenk, R., 1979a, Freshwater triclads (Turbellaria) of North America. XI. Phagocata holleri, new species, from a cave in North Carolina: Proceedings of the Biological Society of Washington, v. 92, p. 389-393.

Kenk, R., 1979b, Freshwater triclads (Turbellaria) of North America. XII. Another new cave planarian from North Carolina: Brimleyana, v. 2, p. 91-96.

Kenk, R., 1984, Freshwater triclads (Turbellaria) of North America. XV. Two new subterranean species from the Appalachian region: Proceedings of the Biological Society of Washington, v. 97, p. 209-216.

Kenk, R., 1987, Freshwater triclads (Turbellaria) of North America. XVI. More on subterranean species of Phagocata of the eastern United States: Proceedings of the Biological Society of Washington, v. 100, p. 664-673.

Kofoid, C.A., 1900, The plankton of Echo River, Mammoth Cave: Transactions of the American Microscopical Society, v. 21, p. 113-126.

LeGrand, H.E., Ratcliffe, J.A., and Finnegan, J.T., 2014, Natural Heritage Program list of rare animal species of North Carolina: Raleigh, North Carolina, North Carolina Natural Heritage Program, Department of Environment and Natural Resources, $162 \mathrm{p}$.

Lewis, J.J., 2000, Five new species of Pseudotremia from caves in the Blue River area of southern Indiana (Diplopoda: Chordeumatida: Cleidogonidae): Myriapodologica, v. 6, p. 55-68.

Lewis, J.J., 2005a, Bioinventory of caves of the Cumberland escarpment area of Tennessee: Technical report. Tennessee Chapter of the Nature Conservancy, Nashville Tennessee, $158 \mathrm{p}$.

Lewis, J.J., 2005b, Six new species of Pseudotremia from caves of the Tennessee Cumberland Plateau: Zootaxa, v. 1080, p. 17-31.

Lewis, J.J., 2009, Eight new cavernicolous species of the milliped genus Pseudotremia (Diplopoda: Chordeumatida: Cleidogonidae): Virginia Museum of Natural History Special Publication, v. 16, p. 171-186.

Lewis, J.J., and Bowman, T.E., 1977, Caecidotea carolinensis, n. sp., the first subterranean water slater from North Carolina (Crustacea: Isopoda: Asellidae): Proceedings of the Biological Society of Washington, v. 90, p. 968-974.

Lewis, J.J., and Reid, J.W., 2007, Patterns and processes of groundwater invasion by copepods in the Interior Low Plateau of the United States: Acta Carsologica, v. 36, p. 279-289.

McAlpine, D.F., and Reynolds, J.W., 1977, Terrestrial Oligochaeta of some New Brunswick caves with remarks on their ecology: Canadian Field-Naturalist, v. 91, p. 360-366

McAlpine, D.F., Reynolds, J.W., Fletcher, T.J., Trecartin, J.L., and Sabine, D.L., 2001, Sparganophilus eiseni (Oligochaeta, Sparganophilidae) in New Brunswick, an addition to the earthworm fauna of Maritime Canada: Megadrilogica, v. 8, p. 53-56.

Miller, B.T., and Niemiller, M.L., 2005, Pseudotriton ruber. Reproduction: Herpetological Review, v. 36, p. 429.

Miller, B.T., and Niemiller, M.L., 2008, Distribution and relative abundance of Tennessee Cave Salamanders (Gyrinophilus palleucus and Gyrinophilus gulolineatus) with an emphasis on Tennessee populations: Herpetological Conservation and Biology, v. 3, p. 1-20.

Miller, B.T., Niemiller, M.L., and Reynolds, R.G., 2008, Observations on egg-laying behavior and interactions among attending female Red Salamanders (Pseudotriton ruber) with comments on the use of caves by this species: Herpetological Conservation and Biology, v. 3, p. $203-210$.

Niemiller, M.L., Glorioso, B.M., Gray, E., Miller, B.T., Jensen, J.B., and Keyes, T., 2006, Pseudotriton ruber ruber. Size and subterranean aggregation: Herpetological Review, v. 37, p. 438.

Niemiller, M.L., and Miller, B.T., 2009, A survey of the cave-associated herpetofauna of the eastern United States with an emphasis on salamanders: in Proceedings of the 15th International Congress of Speleology, Kerrville, Texas, p. 249-256.

Niemiller, M.L., Osbourn, M.S., Fenolio, D.B., Pauley, T.K., Miller, B.T., and Holsinger, J.R., 2010, Conservation status and habitat use of the West Virginia spring salamander (Gyrinophilus subterraneus) and spring salamander (G. porphyriticus) in General Davis Cave, Greenbrier co., West Virginia: Herpetological Conservation and Biology, v. 5, p. 32-43.

Niemiller, M.L., and Reeves, W.C., 2014, Predation by the stygophilic crayfish Cambarus tenebrosus on the salamander Pseudotriton ruber within a cave in Cannon County, Tennessee, USA: Speleobiology Notes, v. 6, p. 8-13. doi:10.5563/spbn.v6i0.62.

Niemiller, M.L., and Reynolds, R.G., eds, 2011, The Amphibians of Tennessee, Knoxville, Tennessee: University of Tennessee Press, 369 p.

Niemiller, M.L., Taylor, S.J., Slay, M.E., and Hobbs III, H.H., 2019, Diversity patterns in the United States and Canada: in Encyclopedia of Caves, 3rd edition (Culver, D.C., White, W.B., and Pipan, T., eds.). Elsevier, p. 163-176.

Niemiller, M.L., and Zigler, K.S., 2013, Patterns of cave biodiversity and endemism in the Appalachians and Interior Plateau of Tennessee, USA: PLOS ONE, v. 8, art. e64177, 17 p. doi:10.1371/journal. pone.0064177.

Niemiller, M.L., Zigler, K.S., and Fenolio, D.B., 2013, Cave Life of TAG: A Guide to Commonly Encountered Species in Tennessee, Alabama and Georgia: Huntsville, Alabama, Biology Section of the National Speleological Society, $45 \mathrm{p}$.

Niemiller, M.L., Zigler, K.S., Stephen, C.D.R., Carter, E.T., Paterson, A.T., Taylor, S.J., and Engel, A.S., 2016, Vertebrate fauna in caves of eastern Tennessee within the Appalachians karst region, USA: Journal of Cave and Karst Studies, v. 78, p. 1-24.

North Carolina Wildlife Resources Commission, 2015, North Carolina Wildlife Action Plan: Raleigh, North Carolina, North Carolina Wildlife Resources Commission, $1298 \mathrm{p}$

North Carolina Wildlife Resources Commission, 2017a, Protected wildlife species of North Carolina: Raleigh, North Carolina, North Carolina Wildlife Resources Commission, 9 p. 
North Carolina Wildlife Resources Commission, 2017b, Final performance report: White-nose syndrome surveillance and bat monitoring in North Carolina: White-nose syndrome grants to states: E-17-5.11.

O'Keefe, J.M., Pettit, J.L., Loeb, S.C., and Stiver, W.H., 2019, White-nose syndrome dramatically altered the summer bat assemblage in a temperate Southern Appalachian forest: Mammalian Biology, v. 98, p. 146-153.

Osbourn, M.S., 2005, The natural history, distribution, and phenotypic variation of cave-dwelling spring salamanders, Gyrinophilus spp. Cope (Plethodontidae), in West Virginia [Master's thesis]: Marshall University, 207 p.

Patton, A., Apodaca, J.J., Corser, J.D., Wilson, C.R., Williams, L.A., Cameron, A.D., and Wake, D.B., 2019, A new green salamander in the Southern Appalachians: Evolutionary implications of Aneides aeneus and implications for management and conservation with the description of a cryptic microendemic species: Copeia, v. 107, p. 748-763.

Pauley, T.K., 1993, Report of the Upland Vertebrates in the New River Gorge National River. Volumes 1-3 (1989-1992). US Department of the Interior, National Park Service.

Peck, S.B., and Lewis, J.J., 1978, Zoogeography and evolution of the subterranean invertebrate faunas of Illinois and southeastern Missouri: National Speleological Society Bulletin, v. 40, p. 39-63.

Petrie, J.S., 1942, Commercial caves: Bulletin of the National Speleological Society, v. 4, p. 58-63.

Poly, W.J., 2001, Nontroglobitic fishes in Bruffey-Hills Creek Cave, West Virginia, and other caves worldwide: Environmental Biology of Fishes, v. 62, p. 73-83.

Reeves, W.K., 2000a, Caecidotea carolinensis (Isopoda: Asellidae): first record of a stygobite from South Carolina: Journal of Cave and Karst Studies, v. 62, p. 18-19.

Reeves, W.K., 2000b, Invertebrate cavernicoles of the Great Smoky Mountains National Park, USA: Journal of the Elisha Mitchell Scientific Society, v. 116, n. 4, p. 334-343.

Reeves, W.K., 2001, Invertebrate and slime mold cavernicoles of Santee Cave, South Carolina, U.S.A.: Proceedings of the Academy of Natural Sciences of Philadelphia, v. 151, p. 81-85.

Reeves, W.K., Jensen, J.B., and Ozier, J.C., 2000, New faunal and fungal records from caves in Georgia, USA: Journal of Cave and Karst Studies, v. 62, p. 169-179.

Reeves, W.K., and Reynolds, J.W., 1999, New records of cave-dwelling earthworms (Oligochaeta: Lumbricidae, Megascolecidae, and Naididae) and other annelids (Aeolosomatida, Branchiobdellida, and Hirudinea) in the southeastern United States, with notes on their ecology: Megadrilogica, v. 7, p. 65-71.

Reid, J.W., 2004, New records and new species of the genus Diacyclops (Crustacea; Copepoda) from subterranean habitats in southern Indiana, USA: Jeffersoniana, v. 12, p. 1-65.

Reynolds, J.W., 1980, The earthworm family Sparganophilidae (Annelida, Oligochaeta) in North America: Megadrilogica, v. 3, p. $189-204$.

Reynolds, J.W., 1994, Earthworms of North Carolina (Oligochaeta: Acanthodrilidae, Komarekionidae, Lumbricidae, Megascolecidae, Ocnerodrilidae and Sparganophilidae): Megadrilogica, v. 5, p. 53-72.

Schmidt, K., 1994, The Mystery Cave expeditions: American Currents, v. 20, p. 26-31.

Schultz, G.A., 1964, Two additional data on terrestrial isopod crustaceans: Ligidium blueridgensis, sp. nov., from Georgia and a North Carolina cave location for Miktoniscus linearis (Patience, 1908): Journal of the Elisha Mitchell Scientific Society, v. 80, p. 90-94.

Shear, W.A., 2003, The milliped family Trichopetalidae, Part 1: Introduction and genera Trigenotyla Causey, Nannopetalum n. gen., and Causeyella n. gen. (Diplopoda: Chordeumatida, Cleidogonoidea): Zootaxa, v. 321, p. 1-36.

Shear, W.A., 2008, Cave millipedes of the United States. VII. New species and records of the genus Pseudotremia Cope. I. Species from West Virginia, USA (Diplopoda, Chordeumatida, Cleidogonidae): Zootaxa, v. 1764, p. 53-65.

Shear, W.A., 2011, Cave millipedes of the United States. X. New species and records of the genus Pseudotremia Cope. 2. Species from Virginia, USA (Diplopoda, Chordeumatida, Cleidogonidae): Zootaxa, v. 3109, p. 1-38.

Shelley, R.M., 2000, Annotated checklist of the millipedes of North Carolina (Arthropoda: Diplopoda), with remarks on the genus Sigmoria Chamberlin (Polydesmida: Xystodesmidae): Journal of the Elisha Mitchell Scientific Society, v. 116, p. 177-205.

Sket, B., 2008, Can we agree on ecological classifications of subterranean animals? Journal of Natural History, v. 42, n. 21-22, p. $1549-1563$. doi:10.1080/00222930801995762.

Slay, M.E., Niemiller, M.L., Taylor, S.J., and Sutton, M., 2016, Cave Life of the Ozarks: A Guide to Commonly Encountered Species in Arkansas, Missouri, and Oklahoma: Huntsville, Alabama: Biology Section of the National Speleological Society, $45 \mathrm{p}$.

Solari, S., 2018a, Myotis leibii. The IUCN Red List of Threatened Species 2018: e.T14172A22055716. http://dx.doi.org/10.2305/IUCN.UK.2018-2. RLTS.T14172A22055716.en. Downloaded on 04 January 2019.

Solari, S., 2018b, Myotis lucifugus. The IUCN Red List of Threatened Species 2018: e.T14176A22056344. http://dx.doi.org/10.2305/IUCN. UK.2018-2.RLTS.T14176A22056344.en. Downloaded on 04 January 2019.

Solari, S., 2018c, Myotis septentrionalis. The IUCN Red List of Threatened Species 2018: e.T14201A22064312. http://dx.doi.org/10.2305/IUCN. UK.2018-2.RLTS.T14201A22064312.en. Downloaded on 04 January 2019.

Solari, S., 2018d, Perimyotis subflavus. The IUCN Red List of Threatened Species 2018: e.T17366A22123514. http://dx.doi.org/10.2305/IUCN. UK.2018-2.RLTS.T17366A22123514.en. Downloaded on 04 January 2019.

Strayer, D.L., and Reid, J.W., 1999, Distribution of hyporheic cyclopoids (Crustacea: Copepoda) in the eastern United States: Archiv fur Hydrobiologie, v. 145, p. 79-92.

Taylor, N., and Mays, J., 2006, The salamanders Eurycea longicauda and Plethodon glutinosus in Gregorys Cave, TN: Monitoring and observations on ecology and natural history: Southeastern Naturalist, v. 5, p. 435-442. doi:10.1656/1528-7092(2006)5[435:TSELAP]2.0. CO;2.

Taylor, S.J., Krejca, J.K., Niemiller, M.L., Dreslik, M.J., and Phillips, C.A., 2015, Life history and demographic differences between cave and surface populations of the western slimy salamander, Plethodon albagula (Caudata: Plethodontidae), in central Texas: Herpetological Conservation and Biology, v. 10, p. 740-752.

Terwilliger, K., 1991, Virginia's endangered species: Proceedings of a symposium: McDonald and Woodward Publishing Co., Blacksburg, Virginia. $672 \mathrm{p}$.

Trauth, S.E., Robison, H.W., and Plummer, M.V., 2004, The Amphibians and Reptiles of Arkansas: Fayetteville, Arkansas: University of Arkansas Press. $421 \mathrm{p}$.

United States Fish and Wildlife Service, 2007, Indiana Bat (Myotis sodalis) draft recovery plan: first revision: United States Fish and Wildlife Service, $258 \mathrm{p}$. 
United States Fish and Wildlife Service, 2015, Endangered and threatened wildlife and plants; threatened species status for the Northern Longeared Bat with 4(d) rule: Federal Register, v. 80, p. 17974-18033.

Vandel, A., 1950, Biospeologica 71: Campagne speologique de C. Bolivar et R Jeannel dans l'Amerique du Nord (1928). Isopodes terrestres recueillis par C. Bolivar et R. Jeannel (1928) et le Dr. Henrot (1946): Arch. Zool. Exper. Gen., v. 87, p. 183-210.

Verant, M.L., Boyles, J.G., Waldrep, Jr., W., Wibbelt, G., and Blehert, D.S., 2012, Temperature-dependent growth of Geomyces destructans, the fungus that causes bat white-nose syndrome: PLOS One, v. 7, e46280.

Wallace, R.L., 1984, Biological survey report of the Great Smoky Mountains National Park caves: Unpublished report to Great Smoky Mountains National Park, $34 \mathrm{p}$.

Wallace, R.L., 2003, Biological survey report of the Great Smoky Mountains National Park caves: Tennessee Caver, v. 2, p. 3-23.

Yeatman, H.C., 1943, Rare cyclopoid copepods from wells in North Carolina: Journal of the Elisha Mitchell Scientific Society, v. 59, p. 27-36. 